\title{
Wärmeanwendungen unter Berücksichtigung der Wärmeregulation - Teil 2
}

\author{
Otto Pechera,b Thomas Zeiger ${ }^{\mathrm{c}}$ Wolfgang Schobersberger ${ }^{\mathrm{d}, \mathrm{e}}$

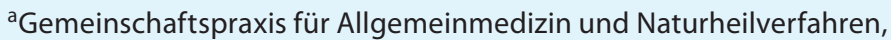 \\ bDropcon CRO, Aying, Deutschland \\ 'Abteilung für Forschung und Entwicklung, Physiotherm, Thaur, \\ dInstitut für Sport-, Alpinmedizin und Gesundheitstourismus (ISAG), Tiroler Landeskrankenanstalten GmbH (TILAK), Innsbruck, \\ eInstitut für Sport-, Alpinmedizin und Gesundheitstourismus (ISAG), UMIT - Private Universität für Gesundheitswissenschaften, \\ Medizinische Informatik und Technik GmbH, Hall in Tirol, Österreich
}

\section{Schlüsselwörter}

Thermoregulation · Thermoneutralzone $\cdot$ Sauna $\cdot$ Infrarotkabine . Infrarotstrahlung · Hauttemperatur · Hauttemperaturmessung · Erythema ab igne $\cdot$ Verbrennung

\section{Zusammenfassung}

Wärmeanwendungen sind in der Krankheitsprävention und zur Linderung vielfältiger Beschwerden ein fester Bestandteil unserer Kultur. Durch die Fortschritte der Medizin wurden Wärmeanwendungen als therapeutisches Verfahren in Randgebiete verdrängt. Mit dem demographischen Wandel, der Zunahme chronischer und vor allem stoffwechselbedingter Erkrankungen sowie durch den Kostendruck im Gesundheitswesen wächst jedoch das Interesse an diesen Verfahren bei Betroffenen sowie auch in der Medizin. Neben der Konduktion und Konvektion werden im privaten und medizinischen Umfeld radiative Verfahren genutzt. Die Wirkung der Wärmeapplikation ist unabhängig vom Medium mit reflektorischen Reaktionen (Muskelentspannung und Schmerzlinderung) sowie Effekten auf Basis der Durchblutungserhöhung und der Temperaturerhöhung im Gewebe erklärbar. Da Wärme im Körper praktisch ausschliesslich mittels Konvektion über das Blut verteilt werden kann, erklärt sich der Einfluss auf das Herzkreislaufsystem. Unter Berücksichtigung der Wärmeregulationsmechanismen lassen sich drei Prinzipien unterscheiden. Die Wärmeapplikation oberhalb, innerhalb und unterhalb der Thermoneutralzone differieren jeweils hinsichtlich der Herzkreislaufbelastung und der Wärmeverteilung. Mit Wärmeanwendungen sind allerdings auch Gefahren verbunden - vor allem, wenn die Wärmeregulation durch Erkrankungen oder Medikationen auf zentraler oder lokaler Ebene gestört ist. Dies geschieht vor allem in Hinblick auf die Herzkreislaufbelastung und auf thermische Schädigungen der Haut. Daher sind Wärmeapplikationssysteme zu fordern, die den Wärmefluss individuell an die Wärmeaufnahmefähigkeit des Körpers anpassen (SensoCare ${ }^{\circledR}$ ). Als Regelgrösse kann dazu die kontinuierliche Messung der Hauteigentemperatur genutzt werden, um eine effiziente und sichere Wärmeanwendung zu ermöglichen.

\section{KARGER}

Fax +497614520714 Information@Karger.com www.karger.com

\section{Keywords}

Thermoregulation - Thermoneutral zone - Sauna · Infrared cabin . Infrared rays · Skin temperature - Skin temperature measurement . Erythema ab igne $\cdot$ Burn injury

\section{Summary}

Thermal Therapies, Taking into Account the Human Thermoregulatory System-Part 2

Thermal therapies in health promotion and alleviation of various diseases are an essential part of our culture. However, advances in medicine have pushed thermal therapies as a therapeutic method to the periphery. The demographic change, the increase of chronic and metabolic diseases, and the rising costs in healthcare are leading once again to a growing interest among both patients and experts in medicine regarding thermal therapies. Beside conduction and convection, the radiative transfer of thermal energy is widely used both in the private and in the medical environment. The effects of heat application, however, are independent from their source. Heat application leads to reflexive reactions (e.g. muscle relaxation and pain relief) as well as effects based on the increasing blood flow and the temperature in the tissue. As heat can only be distributed in the body by convection via the blood, effects on the cardiovascular system can be easily explained. Regarding the human thermoregulatory system, three principles of heat application can be distinguished. Heat application to a human body can be applied in an environment above, within, or below the thermal neutral zone. In each of those cases, both the heat distribution and the resulting cardiovascular burden differ, especially since diseases or medication affect the thermoregulatory system at a central or local level. This may result in a higher risk of cardiovascular burden and thermal damage to the skin. As a result, heat delivery systems should allow an adjustment of the heat flux to the individual heat capacity of the skin and body (SensoCare ${ }^{\circledR}$ ). As a control variable regarding this aspect, a continuous measurement of skin temperature provides the necessary information to establish an effective and safe thermal therapy.

Dr. med. Otto Pecher

Dropcon CRO

Höhenkirchner Strasse 6f, 85653 Aying, Deutschland

pecher@dropcon.de 


\section{Mots clés}

Thermorégulation · Zone thermoneutre · Sauna · Cabine infrarouge ·

Rayonnement infrarouge $\cdot$ Température corporelle $\cdot$ Mesure de la

température corporelle $\cdot$ Érythème $a b$ igne $\cdot$ Brûlure

\section{Résumé}

Applications de chaleur avec régulation thermique - Partie 2

Les applications de chaleur utilisées dans la prévention des maladies et pour soulager diverses douleurs font partie intégrante de notre culture. Avec les progrès de la médecine, les applications de chaleur en tant que procédé thérapeutique ont été tout simplement oubliées. Avec l'évolution démographique, l'augmentation des maladies chroniques et surtout métaboliques, mais aussi en raison de la pression des coûts du système de santé, l'intérêt pour ce procédé croît auprès des personnes concernées et dans la médecine également. En plus de la conduction et de la convection, des procédés de rayonnement sont utilisés dans le milieu privé et médical. Les effets de l'application de chaleur peuvent s'expliquer indépendamment du support avec réactions réfléchissantes (détente des muscles et soulagement de la douleur) et des effets basés sur l'augmentation de la circulation sanguine et de la température dans les tissus. La chaleur dans le corps peut être diffusée dans le sang presque exclusivement par convection, d'où l'influence sur le système cardiovasculaire. En prenant en considération les mécanismes de régulation thermique, on distingue trois principes. L'application de chaleur au-dessus, à l'intérieur et en-dessous de la zone thermoneutre est différente en ce qui concerne la charge du système cardiovasculaire et la diffusion de la chaleur. Les applications de chaleur représentent toutefois des risques également, surtout lorsque la régulation thermique est perturbée sur le plan central ou local par des maladies ou des médicaments. Cela se produit surtout compte tenu de la charge du système cardiovasculaire et des dommages thermiques sur la peau. Par conséquent, il faut exiger des systèmes d'application de chaleur qui adaptent le flux de chaleur individuellement à la capacité d'absorption de chaleur du corps (SensoCare ${ }^{\circledR}$ ). La mesure continue de la température propre de la peau peut être utilisée en tant que grandeur réglée pour permettre une application de chaleur efficace et sûre.

\section{Wärmeanwendungen}

Wärme kann mit dem Ziel einer lokalen bzw. lokoregionalen Temperaturerhöhung im Gewebe oder einer Überwärmung des gesamten Körpers (Verschiebung der Isothermen in der Körperschale bzw. im Körperkern) appliziert werden. Bei Ganzkörperanwendungen muss zwischen subfebrilen und febrilen Temperaturen (über $38^{\circ} \mathrm{C}$ ) unterschieden werden. Künstliches Fieber (Hyperthermie) sollte spezialisierten Einrichtungen vorbehalten bleiben [1-3].

Aus den biochemischen und physiologischen Effekten von Temperaturerhöhungen sowie den komplexen Wärme- regulationsmechanismen und Wärmeabwehrreaktionen lassen sich für die therapeutische Nutzung von Wärme Thesen hinsichtlich der Wirkung, der Einsatzgebiete, der Applikationsform sowie der Effizienz und Sicherheit ableiten. $\mathrm{Zu}$ berücksichtigen sind thermische Schädigungen von Haut und Gewebe sowie Herzkreislaufbelastungen.

\section{Wärmewirkungen}

Für die Wärmewirkungen lassen sich vereinfacht und allgemein folgende, teils überschneidende Bereiche zusammenfassen:

- Ein Wärmereiz führt reflektorisch zur Tonusminderung der Muskulatur, Erhöhung der lokalen Durchblutung, Schmerzlinderung und psychischen Entspannung.

- Über kutiviszerale Reflexe, ausgelöst durch den Wechsel von Wärme- und Kälteanwendungen, können Organe und Muskulatur beeinflusst werden.

- Die vielfältigen Effekte einer «Gewebeerwärmung» lassen sich kursorisch zusammenfassen: Verbesserung der Durchblutung und Mikrozirkulation, Permeabilitätserhöhung der Zellmembranen, Anregung des Stoffwechsels (van't Hoff), Verlängerung der Diffusionsstrecken und Verbesserung der Gewebeversorgung, Stabilisierung der Immunfunktion systemisch und lokal (Schleimhäute) $[1,4,5]$. Hinsichtlich der unspezifischen lokalen Abwehr sind auch die Effekte auf die lokalen Defensin-Konzentrationen von Interesse [6, 7].

- Der Wärmetransport und die Wärmeverteilung erfordern eine sich aktiv anpassende Durchblutung. Dieser Effekt trainiert das Gefässsystem, wodurch eine Stabilisierung der Blutdruckregulation sowie Stabilisierung und Verbesserung der Wärmeregulation möglich ist $[8,9]$.

- Aus naturheilkundlicher Sicht $[10,11]$ werden Ganzkörperwärmeanwendungen $\mathrm{zu}$ den diaphoretischen, ausleitenden Verfahren gerechnet [12]. Die deutliche Erhöhung der Gewebeperfusion verbunden mit der Stoffwechselanregung erreicht im Gewebe (Interzellularraum) eine Mobilisierung von Stoffwechselprodukten und eine Alkalisierung auf Blut-pH-Niveau. Über den Schweiss, der in seiner Zusammensetzung dem Harn ähnelt, kann auch die Ausscheidung verschiedener Schadstoffe unterstützt werden [13].

Die Erhöhung von Mikrozirkulation, Diffusionsstrecke und Umsatzrate wirkt sich auf den gesamten Stoffwechsel in der Zielregion aus. Unterhalb des Fieberbereichs $\left(38^{\circ} \mathrm{C}\right)$ können dabei relativ unspezifische Auswirkungen - auf den Stoffwechsel allgemein - angenommen werden, die in Richtung der physiologischen Homöostase arbeiten und so regulierend auf komplexe Stoffwechselsysteme wirken [1, $14,15]$. 
Oberhalb von $38,5^{\circ} \mathrm{C}$ treten dagegen zunehmend spezifische Effekte (unterschiedliche Aktivitätsmaxima der Enzymsysteme, Heat-Shock-Proteine usw.) in den Vordergrund.

\section{Thermische Belastung der Haut - Wärmeabwehrreaktion}

Wärme kann dem Körper über Konduktion, Konvektion und/oder Radiation zugeführt werden. Eine strikte Trennung der Übertragungswege ist in der Praxis nicht gegeben.

In der Haut erfolgt der Wärmetransport von der äusseren Haut zu den kutanen Blutgefässen und umgekehrt durch direkte Wärmeleitung. Im Körper kommt dagegen praktisch ausschliesslich der konvektive Transport über das Blut zum Tragen [16-18]. Bis auf wenige Ausnahmen (bestimmte Radiowellenfrequenzen) ist also immer die Haut die Applikations- bzw. Austauschfläche für «Wärme», verbunden mit der Gefahr der thermischen Überlastung $[4,14,19-23]$.

Üblicherweise setzt zwischen 43 und $46{ }^{\circ} \mathrm{C}$ der Hitzeschmerz ein. Durch Medikationen (Schmerzmittel, Psychopharmaka, vasoaktive Substanzen usw.), Nervenläsionen, metabolische Erkrankungen, äussere Reize (Reiben der Haut, kalte Haut, Reizanwendungen), psychische Affektionen oder Gewöhnung kann die Sensorik beeinträchtigt sein. Chronische oder akute thermische Schädigungen der Haut - unabhängig von der Art der Wärmeanwendung - sind daher auch im Heim- und Wellnessbereich keine seltene Komplikation [24, 25].

Abbildung 1 zeigt die maximale Expositionszeit in $\mathrm{Ab}$ hängigkeit von der jeweils erreichten Hauteigentemperatur. $43^{\circ} \mathrm{C}$ toleriert die Haut über 8 h. Jede Temperatursteigerung um $0,9^{\circ} \mathrm{C}$ halbiert die Toleranzzeit. Eine Eigentemperatur von $50^{\circ} \mathrm{C}$ führt schon in weniger als $2 \mathrm{~min} \mathrm{zu}$ einer thermischen Schädigung. Bei einer Anwendungsdauer von 40 min sollte die Hauteigentemperatur daher ca. $46^{\circ} \mathrm{C}$ nicht übersteigen [26-28].

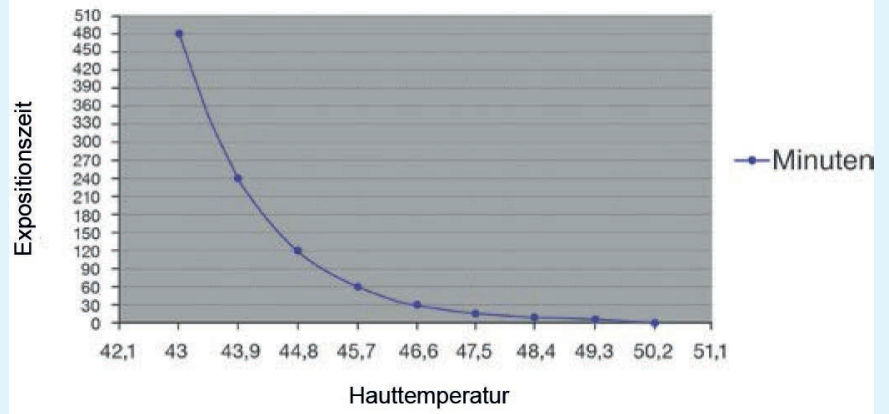

Abb. 1. Hautschäden in Abhängigkeit von der Expositionszeit und Hauttemperatur (modifiziert nach [28]).
Die Höhe der Hauteigentemperatur ist eine Funktion aus dem Wärmezufluss pro Fläche und der Effizienz der lokalen Wärmeabwehrreaktion. Initial kann die zugeführte Wärme nur durch eine Erhöhung der Hautdurchblutung abgeführt werden. Über welchen Übertragungsweg die Temperatur in der Zielregion erreicht wird, ist dabei nicht von Bedeutung.

Die Wärmeabwehrreaktion durch Konduktion, Konvektion und Radiation der Haut wird aber in unterschiedlichem Ausmass beeinträchtigt. Ferner sind individuelle Gegebenheiten (chronische oder akute Erkrankungen, Hautveränderungen, Medikamente usw.) beim Patienten zu berücksichtigen.

- Kritisch sind Störungen der Wärmeabwehr der Haut auf lokaler und/oder zentraler Ebene, da nicht genügend Wärme abgeführt werden kann. In der Regel unproblematische Wärmeanwendungen können dann zu thermischen Schädigungen der Haut führen.

Die wesentliche und initiale Wärmeabwehr der Haut ist ihre direkte Kühlung durch eine Erhöhung der lokalen Durchblutung [29]. Ihre Beeinträchtigung erhöht das Risiko thermischer Schädigung massiv [30]. Zu berücksichtigen sind hier vor allem mechanischer Druck, adrenerge Reize (Kälte, extreme Hitze, Nervosität), Medikamente und Drogen (Beeinflussung der Gefässreaktion), grossflächige Narben (verminderte Vaskularisierung) sowie Grunderkrankungen mit Polyneuropathie oder Nerven- und Rückenmarksläsionen [31, 32]. Untersuchungen bei Querschnittsverletzungen im oberen BWS-Bereich zeigten, dass die Hauteigentemperatur durch eine Infrarotbestrahlung (IR-C ca. $70 \mathrm{~mW}$ / $\mathrm{cm}^{2}$ Haut) des Rückens unterhalb der Verletzung schnell auf $48^{\circ} \mathrm{C}$ ansteigen kann. Oberhalb der Verletzung pendelte sich die Hauteigentemperatur dagegen meist auf $41^{\circ} \mathrm{C}$ ein [31].

- Kritisch ist ein zu hoher Wärmezufluss, der die Kapazität der Wärmeabwehrreaktion überlastet. Der Wärmezufluss wird durch die Temperaturdifferenz und die Wärmeleitzahl bzw. durch die Bestrahlungsstärke unter Berücksichtigung von Reflexion und Absorption bestimmt $[14,18]$.

Für Infrarotexpositionen werden im Arbeitsschutz unabhängig vom Spektrum $355 \mathrm{~mW} / \mathrm{cm}^{2}$ Haut als 10 -Sekunden-Grenzwert angegeben. Dabei wird vorausgesetzt, dass ein Gesunder eine Reaktionszeit von $10 \mathrm{~s}$ hat, um sich aus der Gefahrensituation zu befreien, ohne Verbrennungen zu erleiden. Für längere Bestrahlungszeiten (Wärmeanwendungen) werden Werte von 80-100 $\mathrm{mW} / \mathrm{cm}^{2}$ Haut als Grenzwert diskutiert [33].

- Kritisch ist ein initial hoher Wärmezufluss (bzw. hohe Bestrahlungsstärke). Der Aufbau der Hautdurchblutung nimmt Zeit in Anspruch und kann durch starke Hitze (adrenerger Reiz) behindert werden. Erst mit zu- 
nehmender Durchblutung steigt die Wärmeaufnahmefähigkeit der Haut.

- Problematisch sind konduktive Wärmeanwendungen, da sie die Wärmeabgabemöglichkeiten (Minderung der Hautdurchblutung durch Druck, Konvektion, Radiation, Evaporation) behindern.

\section{Verbrennungen}

Hautrötungen infolge eines Wärmereizes werden als "flush» bezeichnet, wenn sie innerhalb von $24 \mathrm{~h}$ wieder völlig abblassen. Nach dieser Zeit ist ein fliessender Übergang zu Verbrennungen ersten Grades zu beobachten [28]. Es sind bereits Proteinveränderungen, Ödeme und leichtere Immunreaktionen nachweisbar. In der Literatur finden sich wenige Hinweise zu Verbrennungen ersten Grades, da diese meist keiner ärztlichen Behandlung bedürfen $[34,35]$. Akzidentielle Verbrennungen zweiten und dritten Grades werden in der Literatur im Zusammenhang mit praktisch allen Formen von Wärmeanwendung beschrieben $[25,36,37]$. Akute thermische Schäden (Verbrennungen) können bei einer Hauteigentemperatur von $48{ }^{\circ} \mathrm{C}$ innerhalb von Minuten auftreten [28].

\section{Erythema ab igne}

Das Erythema ab igne (EAI; Feuermelanose) ist eine verzögerte Hautreaktion auf wiederholte Hitzeexpositionen $[24,38]$. Es imponiert als eine fleckige, netzartige, mehr oder minder stark pigmentierte retikuläre Hautzeichnung. Histologisch sind atrophische Veränderungen und immunologische Reaktionen im Sinne einer Photodermatose/Keratose nachweisbar [39]. Das EAI, dessen Bedeutung nicht eindeutig geklärt ist, wird in den letzten Jahren wieder häufiger beobachtet. Es besteht der Verdacht, dass eine über Jahre bestehende Feuermelanose zu malignen Veränderungen (Plattenepithelkarzinome, Basaliome) führen kann [40, 41]. Durch die Vermeidung direkter Wärmeexpositionen von betroffenen Hautarealen erfolgt innerhalb von Wochen bis Monaten eine völlige Abheilung. Zusätzliche therapeutische Massnahmen sind in der Regel nicht erforderlich.

Die Art der Wärmequelle ist von untergeordneter Bedeutung. Beschrieben wurde das EAI im Zusammenhang mit Wärmflaschen [42], Heizkissen [43, 44], Wärmegürteln [45], Popcorn [46], Sitzheizungen [47], Laptops [48], heissen Wasserbädern [49], Sauna [25] sowie natürlicher und artifizieller Infrarotstrahlung [50-52].

Das Auftreten des EAI korreliert mit der Häufigkeit und Dauer der Hitzeexposition $[53,54]$ sowie der erreichten Hauteigentemperatur [27]. Bei wiederholter Exposition wird die Schwelle schon bei $43^{\circ} \mathrm{C}$ gesehen $[26,28,55]$.

Eine pathophysiologische Gemeinsamkeit kann eine Beeinträchtigung der Wärmeabwehrreaktion (Durchblutung, Wärmeabgabe) der Haut sein. Gestützt wird diese
Spekulation durch das deutlich seltenere Vorkommen des EAI im Zusammenhang mit Infrarotbestrahlungen, die die Wärmeabwehrmechanismen der Haut am wenigsten beeinträchtigen.

\section{Konduktive Wärmeapplikation}

Die konduktive Wärmezufuhr erfordert einen direkten Kontakt und kann die Durchblutung der Haut und damit ihre initiale Kühlung behindern. Ferner wird die lokale Wärmeabgabe der Haut (Konduktion, Konvektion, Radiation und Verdunstung) blockiert. Eine Kühlung der Haut kann allein über eine Erhöhung der lokalen Durchblutung erfolgen.

Bei direkten Wärmeanwendungen (Heizkissen, Wärmflasche, Kirschkernkissen, Lavasandkissen usw.) kann durch eine entsprechende Ausbildung der Oberflächen (Riffelung, Frotteeüberzug) oder Zwischenlagen (z.B. Fango) eine stehende Luftschicht erzeugt werden, die als guter Isolator die Gefahr einer thermischen Schädigung minimiert.

Aus Sicherheitsgründen sollte die Temperatur des passiven Mediums $46{ }^{\circ} \mathrm{C}$ nicht übersteigen. Selbst wenn die lokalen Wärmeabwehrmechanismen der Haut nicht wirksam werden (mangelnde Hautdurchblutung), ist dann eine thermische Schädigung der Haut bei üblichen Anwendungszeiten nicht anzunehmen.

In der Balneo- und Hydrotherapie werden Temperaturen von bis zu $50{ }^{\circ} \mathrm{C}$ für feuchte Wärmeanwendungen durch Peloide als tolerabel angegeben. Dies setzt allerdings voraus, dass die verwendeten Medien eine geringe Wärmeleitzahl aufweisen. Je höher die Wärmeleitzahl ist, desto höher ist der Wärmefluss. Die Durchblutung der Haut reicht dann nicht mehr aus, um den Wärmezustrom zu kompensieren.

Feuchte Wärmeapplikationen beeinflussen zusätzlich die Permeabilität der Haut. Verschiedene Publikationen weisen darauf hin, dass wesentliche gesundheitliche Effekte über die Inhaltsstoffe des Mediums (z.B. Phythohormone, Mineralstoffe) erzielt werden können.

\section{Konvektive Wärmeapplikation}

Die konvektive Wärmeapplikation (bewegte Luft oder andere gasförmige Medien, bewegtes Wasser, seltener Öle) weist einige Nachteile der Konduktion nicht bzw. in geringerem Umfang auf.

Der konvektive Wärmeübertrag ist von der Bewegung des verwendeten Mediums und seiner Wärmeleitzahl abhängig. Im Gegensatz zur Luft erreicht die Hauteigentemperatur im umströmenden Wasser näherungsweise die Wassertemperatur. Eine Mischform zwischen konduktivem und konvektivem Wärmetransport ist das Bad in 
stehendem Wasser. Die Wärmeabwehrmechanismen sind bis auf den Kopf (Vollbad) blockiert.

Der konvektive Wärmeübertrag durch erhitzte Luft hängt wesentlich von der Luftfeuchte und dem Luftdruck ab. Letzteres ist aber nur im Umfeld hyperbarer Anwendungen zu berücksichtigen. Je höher die Luftfeuchtigkeit, desto höher ist der Wärmeeintrag aufgrund der höheren Wärmeleitfähigkeit des Wasserdampfs. Zudem steigt mit der Lufttemperatur und der Luftfeuchtigkeit der Dampfdruck, sodass zunehmend die Wärmeabgabe durch Evaporation blockiert wird.

\section{Radiation - Elektromagnetische Strahlung}

Bei Wärmeapplikationen über elektromagnetische Strahlung bleiben die Wärmeabwehrreaktionen (Durchblutung) und die Wärmeabgabemechanismen (Konduktion, Konvektion, Infrarot-C-Abstrahlung, Evaporation) weitgehend unbeeinflusst. Geeignet sind die Wellenlängen des Infrarotbereichs und bestimmter Anteile des Radiowellenbereichs $[19,56]$. Während das gesamte Infrarotspektrum in der Haut absorbiert und in Wärme umgewandelt wird, erreichen bestimmte Radiofrequenzen Eindringtiefen von mehreren Zentimetern. Diese Radiofrequenzen bergen jedoch höhere Risiken einer thermischen Gewebeschädigung, da dem Menschen im Gewebe das natürliche Sensorium für Hitze fehlt [20].

Elektromagnetische Strahlung entsteht auf vielfältige Weise, z.B. infolge von Kernreaktionen, Quantensprüngen von Elektronen, bewegten elektrischen Ladungen oder beliebigen Fluktuationen in einem elektromagnetischen Feld. Ihre Eigenschaften werden durch das Wellen- und das Teilchenmodell beschrieben, die in der Quantenphysik zusammengeführt werden.

Die Wellen bzw. Photonen bewegen sich definitionsgemäss mit Lichtgeschwindigkeit und haben keine Ruhemasse. Die Energie ist proportional der Schwingungsfrequenz. Je höher die Frequenz, desto energiereicher ist die Strahlung (Tab. 1).

Tab. 1. Unterteilung der elektromagnetischen Strahlung (modifiziert nach [57])

\begin{tabular}{ll}
\hline Ionisierende Strahlung & \\
Kosmische Strahlung & $10^{-16}$ bis $10^{-12} \mathrm{~m}$ \\
Gammastrahlung & $10^{-14}$ bis $10^{-12} \mathrm{~m}$ \\
Röntgenstrahlung & $10^{-12}$ bis $10^{-7} \mathrm{~m}$ \\
Kurzwellige UV-Strahlung & $10^{-7}$ bis $1,8 \times 10^{-7} \mathrm{~m}$ \\
Optische Strahlung & \\
Mittel- und langwellige UV-Strahlung & $1,8 \times 10^{-7} \mathrm{bis} 3,8 \times 10^{-7} \mathrm{~m}$ \\
Sichtbares Licht & $3,8 \times 10^{-7} \mathrm{bis} 7,8 \times 10^{-7} \mathrm{~m}$ \\
Infrarotstrahlung & $7,8 \times 10^{-7} \mathrm{bis} 10^{-5} \mathrm{~m}$ \\
Hochfrequenzstrahlung & \\
Terahertz-Wellen (300 GHz - 10 THz) & $10^{-5}$ bis $10^{-3} \mathrm{~m}$ \\
Mikrowellen (300 MHz bis 300 GHz), & $10^{-3}$ bis $10^{-1} \mathrm{~m}$ \\
$\quad$ Radartechnik, Mikrowellenherd, Mobilfunk, & \\
$\quad$ Satellitenfernsehen, Funk, WLAN, Bluetooth & \\
Radiowellen & $10^{-1}$ bis $10^{6} \mathrm{~m}$ \\
\hline
\end{tabular}

Wärmeanwendungen und Wärmeregulation

\section{Wechselwirkung mit Materie}

In Abhängigkeit von ihrer Energie tritt elektromagnetische Strahlung auf unterschiedliche Weise in Erscheinung und entfaltet unterschiedliche Wirkungen auf Materie und Leben.

Ist die Energie grösser als die Bindungsenergie in den Atomen (im Allgemeinen $>5$ Elektronenvolt (eV)), spricht man von ionisierender elektromagnetischer Strahlung. Diese Photonen können Elektronen aus den Atomschalen schlagen und z.B. das Genom direkt verändern. Ferner entstehen Ionen, die weitere Reaktionen auslösen und so Zellorganellen und Genom schädigen können.

Unterhalb von $5 \mathrm{eV}$ reicht die Energie für eine Ionisierung im Allgemeinen nicht mehr aus. Die physiologischen Wirkungen der «nicht ionisierenden» elektromagnetischen Strahlung (langwellige UV-Strahlung, sichtbares Licht, Infrarot, Terahertz-Wellen, Mikrowelle, Radar, Wechselstrom usw.) auf den Menschen abhängig von der Bestrahlungsstärke und der Häufigkeit der Exposition - werden teilweise kontrovers diskutiert [58].

Weisses sichtbares Licht wird zur Behandlung der saisonal bedingten Depression und auch von endogenen Formen eingesetzt [59]. Kurzwelliges sichtbares Licht (blau) hemmt, langwelliges rotes Licht fördert dagegen die Melatonin-Produktion mit den entsprechenden Auswirkungen auf den Schlaf [60]. Blaues Licht hemmt entzündliche Reaktionen, rotes Licht erhöht die Mikrozirkulation [61, 62]. Die Mobilfunkstrahlung wurde als potenziell kanzerogener Faktor eingestuft [63]. Auch bezüglich Radarstrahlung wird eine Promotion von Krebserkrankungen diskutiert [64, 65].

Die Anwendung elektromagnetischer Strahlung zur Wärmeapplikation ist weit verbreitet. Neben der Hochfrequenzstrahlung wird im medizinischen Umfeld, aber vor allem im Heim-, Spa,- und Wellnessbereich überwiegend Infrarotstrahlung eingesetzt.

\section{Hochfrequenzstrahlung zur Wärmeapplikation}

Elektromagnetische Strahlung im Megahertz(MHz)und Gigahertz $(\mathrm{GHz})$-Bereich wird vor allem zur lokalen bzw. regionalen Wärmeapplikation eingesetzt. Dadurch soll die thermische Belastung der Haut sowie des Unterhautfettgewebes gering gehalten und eine direkte Tiefenerwärmung erreicht werden [66, 67].

Kurzwellen bzw. Radiowellen mit Frequenzen von 8-30 MHz werden zur Überwärmung tiefer liegender Tumoren, aber auch in der Physiotherapie zur Erwärmung von Muskelgewebe eingesetzt. Die Strahlung dringt mit $5-10 \mathrm{~cm}$ tiefer als höherfrequente Wellen in das Gewebe ein. Gewebe mit niedrigerem Wassergehalt wird weniger stark erwärmt [68]. Mit dieser Technik kann im behandelten Gebiet eine relativ gleichmässige Wärmeverteilung erreicht werden. 
Vor allem in Hinblick auf die regionale Hyperthermie zur Behandlung von Tumoren werden «nicht thermische» - direkt zytostatische - Effekte bestimmter Frequenzen diskutiert. Als Grund hierfür wird angegeben, dass die Dielektrizitätskonstante im Tumorgewebe 10 - bis 30 -mal höher liegt, die elektrische Leitfähigkeit sogar um den Faktor 100.

Zur regionalen Hyperthermie mittels Dezimeterwellen wird mit Frequenzen von 400-920 MHz gearbeitet. Dabei werden mehrere Applikatoren so geschaltet, dass die Temperatur im Tumorgewebe durch Phasenverschiebungen gezielt erhöht (Fokussierung) werden kann. Mit steigender Frequenz verringert sich die Eindringtiefe und die Inhomogenität der Temperaturverteilung im Gewebe nimmt zu. Die Gefahr von Hotspots steigt.

Auch zur gezielten Tiefenerwärmung der Muskeln wird elektromagnetische Strahlung im MHz-Bereich verwendet. Bei 433,92 MHz/69 cm soll dabei im wasserreichen Muskelgewebe die stärkste Absorption möglich sein.

Der Mikrowellenbereich wird wiederum vor allem in der Onkologie zur thermischen Behandlung oberflächlicher Tumoren eingesetzt. Die Frequenzen liegen im GHzBereich, mit einer geringen Eindringtiefe. Aufgrund der inhomogenen Feldverteilung und unspezifischen Kopplungen ist die Gefahr der Erzeugung von Hotspots gross.

\section{Infrarotstrahlung}

Jeder Körper gibt entsprechend seiner Oberflächentemperatur ein Spektrum elektromagnetischer Strahlung ab, das je nach Höhe der Temperatur von der Gammastrahlung bis zum Radiowellenbereich reichen kann.

Infrarotstrahlung ist somit nur ein kleiner Teil des gesamten elektromagnetischen Spektrums. Die heute gebräuchlichen Einteilungen des Infrarotspektrums sind nicht einheitlich und basieren nicht auf physiologischen Wirkungen am Menschen [57]:

- Infrarot A: Kurzwellige Infrarotstrahlung

(780-1400 nm);

- Infrarot B: Mittelwellige Infrarotstrahlung

$(1400-3000 \mathrm{~nm})$;

- Infrarot C: Langwellige Infrarotstrahlung

(3000 bis 1 Million $\mathrm{nm}$ );

- Terahertzstrahlung (neu): (30 000 bis 1 Million nm).

In Publikationen findet sich häufig auch die Unterteilung in «nahe» $(750-3000 \mathrm{~nm})$, «mittlere» (3000$30000 \mathrm{~nm}$ ) und «ferne» (30 000 bis 1 Million $\mathrm{nm}$ ) Infrarotstrahlung [69]. Die unterschiedlichen Einteilungen erschweren häufig den Vergleich publizierter Daten.

Bei einer Wellenlänge von $780 \mathrm{~nm}$ liegt die Energie der Photonen $(1,59 \mathrm{eV})$ weit unterhalb der Ionisierungsenergie. Das Besondere des Frequenzbereichs der Infrarotstrahlung ist, dass er energetisch mit den Eigenschwingungen von Materie korrespondiert. Bei korrespondierenden Wellenlängen erfolgt der Energieübertrag effizienter. Das charakteristische Absorptions- und Reflexionsspektrum im Infrarotbereich lässt sich für chemische Analysen [57] und möglicherweise auch in der Diagnostik [70] nutzen.

Die absorbierte Energie der Infrarotstrahlung wird in Schwingungsenergie der Materie - als Wärme «spürbar» - umgewandelt. Die Begriffe «Infrarotstrahlung» und «Wärmestrahlung» werden umgangssprachlich daher synonym verwendet.

Grenzwerte für Infrarotbestrahlungen: Bezüglich der Bestrahlungsgrenzwerte für Anwendungen am Menschen unterscheiden weder die International Commission on Non-Ionizing Radiation Protection (ICNIRP) [33] noch die 2010 in Kraft getretene ÖNORM [71, 71a] zwischen den Spektralbereichen. Für die Anwendung am Menschen liegen die Grenzwerte bei $100 \mathrm{~mW} / \mathrm{cm}^{2}$ (Haut) und $10 \mathrm{~mW} /$ $\mathrm{cm}^{2}$ (Auge) [33].

Strahler: Mit Ausnahme von Lasern und Dioden emittieren Oberflächen immer ein breites Spektrum an elektromagnetischer Strahlung. Die Verteilung der Wellenlängen und deren Intensität werden in Abhängigkeit von der Oberflächentemperatur durch das Strahlungsgesetz von Planck beschrieben. Das Wiensche Verschiebungsgesetz beschreibt die Lage des Maximums im jeweils abgegebenen Spektrum. Der Aufbau des Strahlers und das Oberflächenmaterial sind dabei sekundär. Für den Einsatz als Wärmequelle sind die physikalischen Zusammenhänge zwischen Oberflächentemperatur und Bestrahlungsstärke sowie Abstand und Bestrahlungsstärke entscheidend.

Der «schwarze Strahler», ein theoretisch-physikalisches Modell, ist das Bezugssystem. Durch eine Verdopplung der Oberflächentemperatur (basierend auf der physikalischen Temperatureinheit «Kelvin») erhöht sich die Bestrahlungsstärke um den Faktor 16. Ferner gilt, dass aus der Verdopplung des Abstands zum Strahler bei gleicher Leistung und gleicher bestrahlter Fläche eine um das Vierfache niedrigere Bestrahlungsstärke resultiert.

Wird die Leistung eines Strahlers reduziert, sinkt dessen Oberflächentemperatur, das abgegebene Spektrum verschiebt sich in den langwelligen Bereich und die Bestrahlungsstärke nimmt deutlich ab. Ein praktisches Beispiel für diesen Zusammenhang ist das Dimmen einer einfachen Glühbirne.

Mit Ausnahme von Lasern und Dioden gibt es daher keine reinen Infrarot-A- oder -B-Strahler.

Natürliche Infrarotstrahlung: Infrarotstrahlung umgibt den Menschen zu jeder Zeit, ubiquitär. Das Abstrahlspektrum der Sonne (Oberflächentemperatur ca. $5500^{\circ} \mathrm{C}$ ) umfasst Gammastrahlung, UV-Strahlung, sichtbares Licht und Infrarotstrahlung bis hin zu den Radiowellen. Das Maximum liegt im grünen, sichtbaren Lichtbereich. Die Feuchtigkeit der Erdatmosphäre absorbiert grosse Anteile von Infrarot B und C. Auf die Erdoberfläche trifft daher ein relativ hoher Infrarot-A-Anteil, der die Materie erwärmt. 
Unsere Umwelt, warme Luft und Objekte, weist in der Regel deutlich niedrigere Oberflächentemperaturen auf. Unterhalb von ca. $300{ }^{\circ} \mathrm{C}$ werden keine Infrarot-A- oder B-Anteile emittiert. Der Mensch ist also die weit überwiegende Zeit Infrarot-C-Strahlung ausgesetzt, die er auch selbst abstrahlt.

Nicht thermische Wirkungen der Infrarotstrahlung: Trotz der geringen Energie der Infrarotstrahlung werden direkte, «nicht thermische» Effekte auf lebende Zellen diskutiert. Verschiedene Autoren sehen Wellenlängen unter $850 \mathrm{~nm}$ als ausreichend an, um photochemische Reaktionen in den Zellen auszulösen [72, 73]. Auch für die «ferne» Infrarotstrahlung werden «nicht thermische» Effekte diskutiert [74]. Hier ist der molekulare Mechanismus noch spekulativ.

Arbeiten, die eine Hautkrebspromotion (Basaliome, Plattenepithelkarzinome) und beschleunigte Hautalterung durch kurzwellige Infrarot-A-Strahlung zu belegen scheinen, werden kontrovers diskutiert, konnten bisher aber nicht widerlegt werden $[51,53,69,75,76]$.

Die beschriebenen Effekte, negative wie positive [77], können allerdings auch durch Temperaturerhöhungen (Radikalbildung, Durchblutung, Stoffwechsel, Perfusion) grösstenteils erklärt werden [26, 27, 55].

Eindringtiefen der Infrarotphotonen: Um eine «Tiefenwirkung» (Wärmeeintrag, Laserakupunktur) zu begründen, wird vielfach über die Hauteindringtiefe bestimmter Frequenzbereiche der Infrarotstrahlung diskutiert.

Das Infrarotspektrum weist Absorptionsminima - gerne als «biologische Fenster» bezeichnet - für Wasser auf. Diese «Fenster» findet man vor allem im kurzwelligen A-, in wesentlich geringerem Umfang aber auch im B- und CBereich. Da die menschliche Haut eine hohe Hydratation aufweist, wird die Strahlung aus diesen Spektralbereichen erst in tieferen Hautschichten absorbiert. 5\% dieser Photo-

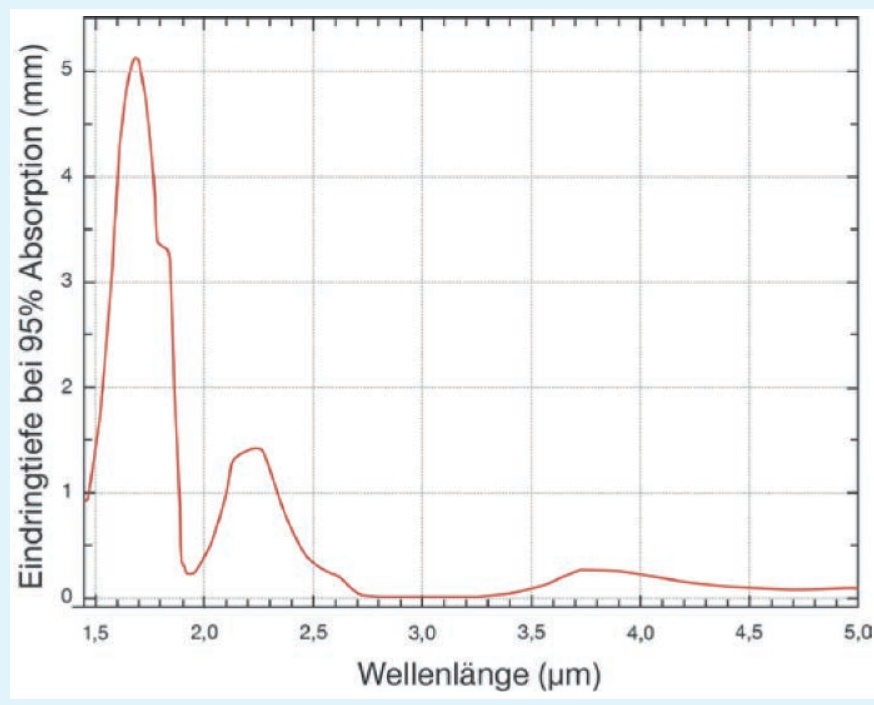

Abb. 2. Hauteindringtiefe (modifiziert nach [81]).

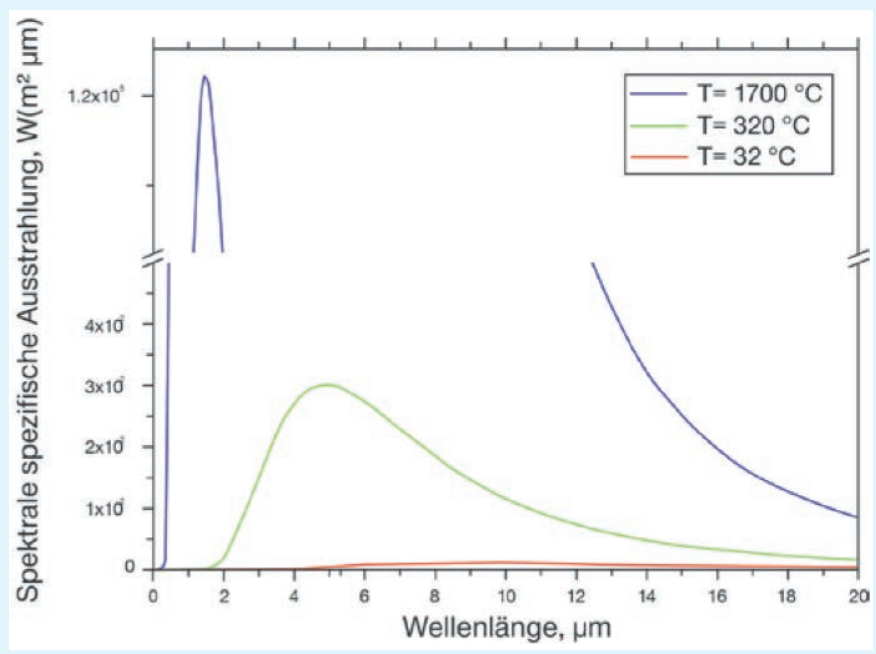

Abb. 3. Spektralverteilung bei verschiedenen Oberflächentemperaturen nach Planck. Da eine Verdopplung der Temperatur näherungsweise eine 16-fach höhere Bestrahlungsstärke ergibt, musste der Graph des Spektrums mit $1700^{\circ} \mathrm{C}$ bei dieser Darstellung unterbrochen werden.

nen können bis zu 4-5 mm in die Haut eindringen. 95\% der Photonen werden in den oberen Hautschichten absorbiert - 75\% innerhalb der ersten 0,75 $\mathrm{mm}$ [78-80].

Abbildung 2 zeigt die Hauteindringtiefen in Abhängigkeit von der Wellenlänge [81]. Diese Darstellung wird von verschiedenen Autoren allerdings angezweifelt [82].

$\mathrm{Da}$ jede Materie ein Infrarotspektrum abstrahlt, stellt die Bestimmung der Eindringtiefe in lebendes Gewebe bisher teils unlösbare Anforderungen an den Versuchsaufbau. Einfacher ist die Messung der Eindringtiefe bestimmter Infrarotwellenlängen (800 und $850 \mathrm{~nm}$ ), die von von Lasern bzw. Dioden emittiert werden. Die eindeutige $\mathrm{Zu}-$ ordenbarkeit der Frequenz erleichtert die Detektion der Infrarotstrahlung im Gewebe. Verschiedene Autoren konnten nur geringe Eindringtiefen feststellen - die Hauptabsorption fand in den Bereichen von 0,4-0,8 mm statt. Eine «Tiefenwirkung» der Infrarotstrahlung wurde daher infrage gestellt $[78,79]$ (Abb. 3). Auch unabhängig davon müssen die Auswirkungen für die Praxis kritisch hinterfragt werden.

Bei einer Abstrahltemperatur (Oberflächentemperatur) von $1700{ }^{\circ} \mathrm{C}$ (hell leuchtend) werden ca. $23 \%$ der Leistung im Infrarot-A-Bereich abgestrahlt. Die restlichen Anteile verteilen sich auf das sichtbare Licht sowie Infrarot B und Infrarot C. Für den Energie- bzw. Wärmeübertrag auf die Haut ist allerdings der gesamte Infrarotbereich entscheidend. Eine Berechnung der Fläche unter der Kurve (Area under the curve (AUC)) zeigt, dass jener Photonenanteil von 5\% aus dem «biologischen Fenster» für den Wärmeübertrag zu vernachlässigen ist. Zudem werden kurzwellige Infrarotanteile (A- und kurzwelliger B-Bereich) durch die Hautpigmente bis zu 50\% reflektiert [83]; die Absorption langwelliger Infrarot-C-Strahlung erfolgt $\mathrm{zu}$ nahezu $100 \%$. 
Das Reflexions- und Absorptionsverhalten der Infrarotstrahlung - und damit auch die Eindringtiefe bestimmter Frequenzen - ändert sich entsprechend den molekularen Komponenten der Haut [84]. Weder die Hydratation der Haut noch deren molekulare Komponenten sind konstant [84-86].

Eine direkte Erwärmung tiefer liegender Gewebeschichten ist mit Infrarotstrahlung nicht zu erreichen [87, 88]. Folgerichtig weisen sowohl die ICNIRP als auch die ÖNORM darauf hin, dass Implantate durch Infrarotstrahlung nicht direkt erhitzt oder anderweitig beeinflusst werden können. Auch in der Onkologie werden zur direkten Erhitzung von tiefer liegendem Tumorgewebe Radiowellen statt Infrarotstrahlen eingesetzt. Häufig kolportierte Bezeichnungen wie z.B. «Tiefenwärme-, Arthrose-, Bronchial- und Prostatastrahler» sind daher irreführend.

Ungeachtet dessen kann die wassergefilterte Infrarotstrahlung deutliche Vorteile bei der Bestrahlung offener und nässender Wunden aufweisen, da ein geringerer Anteil der applizierten Strahlung die Wundflüssigkeit erhitzt und die Konduktion so vermindert wird.

Direkte Wärmeleitung in das Gewebe: Die Bestimmung eines Gewebetemperaturgradienten oder einer Durchblutungserhöhung unter Infrarotbestrahlung stösst im Versuchsaufbau ebenfalls an Grenzen. Die Ergebnisse der artifiziellen Situation lassen sich kaum auf die Praxis übertragen [32, 89, 89a]. Die Untersuchungen mussten zwangsläufig am tiefnarkotisierten Tier durchgeführt werden. Tiefe Narkosen setzen die normale Wärmeregulation ausser Kraft, sodass die Daten zu den gemessenen Temperaturgradienten nicht auf den «normalen Anwender» übertragbar sind.

Die Wärmeleitung in der Haut - von den Blutgefässen zur äusseren Haut (Abgabe) und umgekehrt (Aufnahme) - erfolgt mittels Konduktion. Dabei ist zu berücksichtigen, dass die Wärmeleitzahl verschiedener Gewebe um den Faktor 2-3 differiert [4]. Der Körperbereich der Wärmeapplikation ist für die Konduktion entscheidend. Der funktionierenden Wärmeabwehrreaktion der Haut durch eine Veränderung der Durchblutungssituation kommt eine weitere, massgebliche Bedeutung zu. Bei erhaltener Wärmeregulation wird der Hauptanteil der Wärme über das Blut abgeführt. Der Aufbau eines Temperaturgradienten in die Tiefe wird dadurch massiv behindert.

Diesen Zusammenhang bestätigen Untersuchungen mit Patienten, die unter einer hohen Querschnittsverletzung leiden. Identische Bestrahlungsstärken $\left(330^{\circ} \mathrm{C}\right.$ Abstrahltemperatur, $80 \mathrm{~mW} / \mathrm{cm}^{2}$ Haut) führten im innervierten Hautbereich, oberhalb der Verletzung, zu einer tolerablen Hauteigentemperatur von $41{ }^{\circ} \mathrm{C}$. Im nicht innervierten Bereich, unterhalb der Verletzung, stieg die Hauteigentemperatur dagegen innerhalb von Minuten auf $48^{\circ} \mathrm{C}$ an, was einen Abbruch der Untersuchung zur Folge hatte [31].

\section{Sicherheitsentwicklung zur Wärmeapplikation}

Jede Wärmeapplikation bietet grundsätzlich die Gefahr einer thermischen Schädigung der Haut. Infrarotbestrahlungen bieten den Vorteil, dass der Wärmeübertrag berührungslos erfolgt und so die Abwehrreaktion am wenigsten beeinträchtigt wird.

Die teils kontroversen Ergebnisse verschiedener Autoren zu Infrarotbestrahlungen erfordern neue Untersuchungen auf Basis eines breiten Konsenses hinsichtlich der Untersuchungsbedingungen und Definitionen [82]. Unabhängig davon muss die Frage gestellt werden, ob Untersuchungen zu Eindringtiefen und nicht thermischen Effekten im Hinblick auf das Ziel «Wärmeapplikation» nur von akademischem Interesse sind.

In der Haut erfolgt die Wärmeleitung durch Konduktion. Unabhängig von den Spektralanteilen kommt es zu einem Temperaturausgleich in der Haut. Die thermische Belastung der Haut ist der limitierende Faktor [28]. Diese sollte während einer Wärmeanwendung zur sicheren Vermeidung akuter (Verbrennung) und chronischer (EAI) thermischer Schädigungen $43^{\circ} \mathrm{C}$ dauerhaft und/oder wiederholt nicht übersteigen. Die übliche Hitzeschmerzreaktion ist - vor allem bei Medikationen sowie akuten und chronischen Erkrankungen - kein zuverlässiger Parameter, um thermische Schäden zu vermeiden.

Zur Einhaltung der Temperaturgrenzwerte ist das $\mathrm{Zu}$ sammenspiel zwischen Wärmefluss (Bestrahlungsstärke) und Wärmeabwehrreaktion (Hautdurchblutung und Wärmeregulation) entscheidend. Der Aufbau einer adäquaten Durchblutung erfordert Zeit und kann durch eine Vielzahl von Faktoren auf lokaler und/oder zentraler Ebene individuell beeinträchtigt sein [71]. Selbst bei einer üblicherweise unproblematischen Wärmeapplikation können daher Hauttemperaturen von deutlich über $46^{\circ} \mathrm{C}$ auftreten, ohne dass der Betroffene adäquat reagieren kann.

Die zu fordernde Sicherheit kann nur durch Wärmeapplikationssysteme erreicht werden, welche die Hauttemperatur im bestrahlten Hautareal kontinuierlich und berührungslos erfassen und den Wärmezufluss auf Basis dieser Werte automatisch regulieren [31]. Entsprechende Systeme, die die Hauttemperatur je nach Anwendung mittels 2-6 Sensoren überwachen und über computergesteuerte Berechnungen (Steigung, Maximalwert) die Bestrahlungsstärke (Infrarot-C) individuell anpassen, stehen seit 2010 zur Verfügung (SensoCare ${ }^{\circledR}$ ).

Die ersten Untersuchungen zeigen, wie unterschiedlich selbst bei jungen, gesunden Probanden die Wärmeaufnahmefähigkeit der Haut ist und zudem tagesabhängig variiert. Die Funktionsfähigkeit und Sicherheit des Systems übertraf in seiner Effizienz und Regelgenauigkeit die Erwartungen.

Die folgenden Grafiken (Abb. 4) veranschaulichen, wie stark die Wärmeabwehrreaktion bei einem Menschen 
mit Rückenmarksverletzung variieren kann. Als Zieltemperatur wurden $43{ }^{\circ} \mathrm{C}$ vorgegeben, die einmal bereits mit ca. $30 \mathrm{~mW}$ erreicht wurden, während an einem anderen Tag dagegen deutlich höhere (bis $90 \mathrm{~mW}$ ) Bestrahlungsstärken appliziert werden konnten.

Mit derartigen Systemen können thermische Überlastungen der Haut unabhängig von der Art der Beein-

a

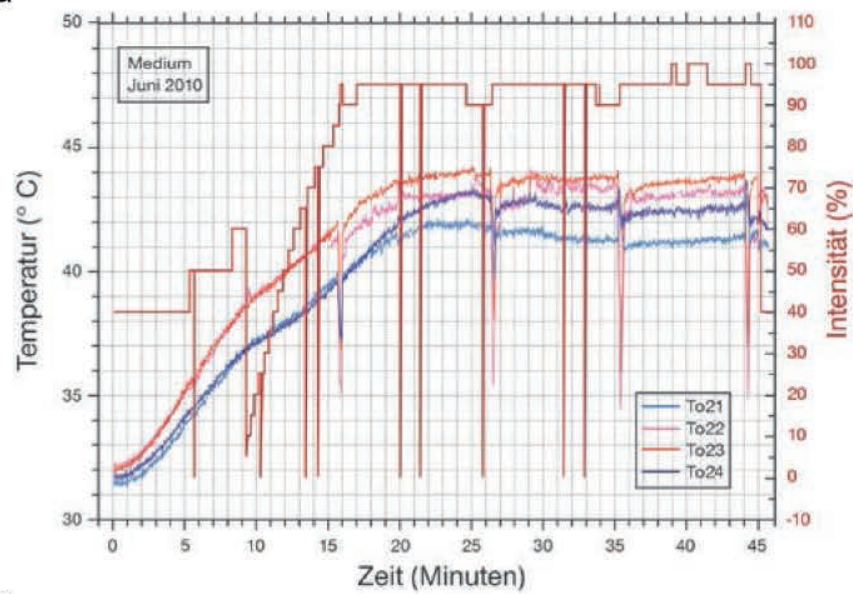

b

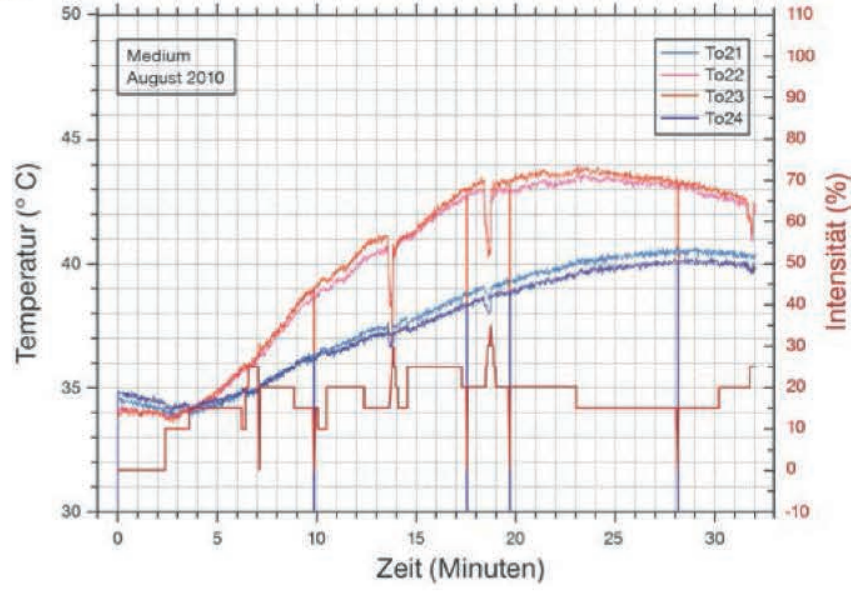

C

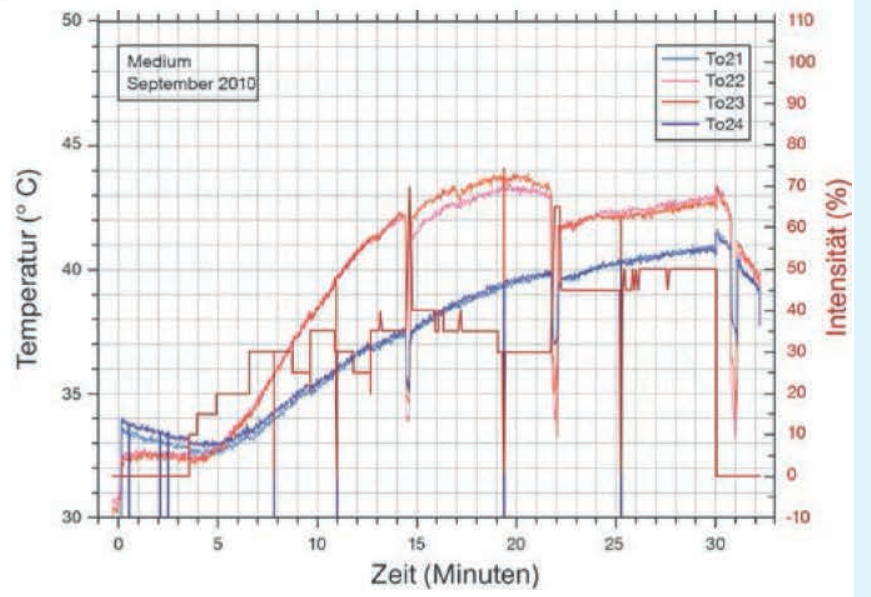

Abb. 4. Hauteigentemperatur in Abhängigkeit von Bestrahlungsstärke und Effektivität der Wärmeregulation (modifiziert nach [31]). a Medium Juni 2010. b Medium August 2010. c Medium September 2010. trächtigung der Wärmeabwehrreaktion ausgeschlossen werden. Die Nutzung der maximalen Wärmeaufnahmefähigkeit gewährleistet gleichzeitig auch einen maximalen Wärmeeintrag bei höchster Anwendungssicherheit (Hautbelastung, Herzkreislaufsystem). Da das System automatisiert arbeitet und weder eine Vorbereitung noch in der Regel eine Begleitung benötigt, ergeben sich im praktischen Einsatz zudem deutliche Kostenvorteile. Daher sollten derartige Systeme generell zum Einsatz kommen [31].

\section{Herzkreislaufbelastungen}

Da Ganzkörperwärmeanwendungen mit dem Ziel erfolgen, im gesamten Körper die Temperatur kontrolliert zu erhöhen, müssen die Wärmeabwehrreaktionen stufenweise überwunden werden. Daher ist der Ablauf der physiologischen Wärmeregulation und die daraus resultierende Herzkreislaufbelastung besonders zu beachten $[4,17$, $68,90]$. Grundsätzlich zu unterscheiden sind Ganzkörperwärmeanwendungen innerhalb oder oberhalb der Thermoneutralzone (TNZ).

Bei Hitze wird die Hautdurchblutung massiv erhöht, um die Haut zu kühlen und gleichzeitig innere Wärme nach aussen abgeben zu können. Nimmt das Blut mehr Wärme auf, als es über die Haut an die Umgebung abgeben kann, erhitzt es sich. Um nun einen schnellen Anstieg der Körperkerntemperatur (KKT) zu vermeiden, muss der Körper den Blutrückstrom aus der Haut zum Körperkern reduzieren $[4,18,91,91 \mathrm{a}]$. Dennoch wird weiter Blut in die Haut transferiert, um die Hautkühlung zu gewährleisten. Im zentralen Kreislauf kommt es zunehmend zu einem Volumenmangel. Die Herzkreislaufbelastung steigt massiv an.

In heissen Umgebungen wirkt die Wärmeregulation also einer beabsichtigten, zentralen Wärmezufuhr entgegen. Vor allem für Ganzkörperwärmeanwendungen gilt: Je massiver gegen die Thermoregulation gearbeitet wird, desto höher sind zwangsläufig die Belastungen für den Organismus $[17,68,92]$.

In der TNZ $\left(27-37^{\circ} \mathrm{C}\right.$ Lufttemperatur bei $50 \%$ Luftfeuchtigkeit) sind dagegen Wärmeabgabe und -aufnahme ausgeglichen. In dieser Situation muss der Körper keine aktive Wärmeregulation betreiben, um die Körpertemperatur in allen Bereichen aufrechtzuerhalten.

\section{Wärmeanwendungen oberhalb \\ der Thermoneutralzone}

\section{Saunaanwendungen}

Saunaanwendungen $\left(80-100^{\circ} \mathrm{C}\right.$ Lufttemperatur) finden oberhalb der TNZ statt. Die Erwärmung der Haut erfolgt 
bei der finnischen Sauna je nach Bauart zu 60-70\% über die erhitzte Luft und zu 30-40\% über Infrarotstrahlung.

Über Konvektion und Infrarotstrahlung wird mehr Wärme aufgenommen, als abgegeben werden kann (siehe oben). Trotz der in der Regel trockenen Luft ist der Dampfdruck höher als jener, den die Haut aufbauen kann. Der abtropfende Schweiss trägt nicht zur Kühlung bei.

Um die thermische Belastung der Haut zu reduzieren, wird die Durchblutung massiv erhöht. Der Blutrückstrom zum Körperinnern wird dagegen reduziert, um einen Anstieg der KKT zu verhindern. Das Herzminutenvolumen steigt massiv an.

Bei Saunaanwendungen beobachtet man anfänglich keine Erhöhung der KKT, gegebenenfalls initial sogar einen leichten Abfall [1, 91, 93-98].

Erst nach einer längeren Expositionszeit (8-15 min) muss der Blutrückstrom aufgrund des relativen, zentralen Volumenmangels wieder etabliert werden. Es kommt zu einem schnellen Anstieg der KKT auf bis zu $39^{\circ} \mathrm{C}$. Diese Situation ist für Menschen mit Herzkreislauferkrankungen und/oder nach Alkoholkonsum ein gesundheitliches Risiko $[25,99]$.

Verschiedene Autoren sehen daher eine 10-minütige Exposition in der finnischen Sauna als Limit, gefolgt von einer Kaltwasseranwendung und einer Ruhephase. Wird die Anwendung rechtzeitig verlassen, bleibt die KKT konstant. Diese Anwendung wird im Sinne einer KneippAnwendung gesehen [97]. Problematisch ist, dass die verträgliche Expositionsdauer individuell und interindividuell deutlich variieren kann. Eine fixe Zeitvorgabe erscheint im Hinblick auf die vorliegenden Daten wenig zielführend.

\section{Biosauna oder Infrarotwärmekabine $\left(50-60^{\circ} \mathrm{C}\right)$ mit/ohne grossflächige Infrarotbestrahlungen}

Ganzkörperwärmeanwendungen bei niedrigeren Temperaturen $\left(50-60^{\circ} \mathrm{C}\right)$ unterschieden sich hinsichtlich der Wärmeregulation nicht prinzipiell von der «Sauna». Die Herzkreislaufbelastung ist aber deutlich geringer. Die Evaporation kann zur Wärmeabgabe genutzt werden.

Im Unterschied zur Biosauna kann bei Infrarotwärmekabinen je nach Bauart (Plattenkabine, Strahler) der Anteil des Wärmeübertrags mittels Infrarotstrahlung auf 60 $80 \%$ erhöht werden. Die «Strahlungswärme» wird als angenehmer empfunden.

Wird die Anwendung rechtzeitig verlassen, kühlt das Blut in der Haut ab und wird schrittweise dem Kreislauf wieder zur Verfügung gestellt. Die KKT bleibt wiederum auf konstantem Niveau.

Korrekt durchgeführt bieten diese «Reizanwendungen» im Kneipp'schen Sinne einen starken vegetativen Stimulus auf verschiedene Organe, ein Gefässtraining sowie eine gute Haut- und Schleimhautdurchblutung mit positiven Auswirkungen (z.B. Erkältungsprophylaxe). Die Schweissbildung unterstützt die Entgiftung vor allem im Bereich der äusseren Körperschale $[97,100$, 101].

Eine gleichmässige Durchwärmung der inneren Körperschale wird nicht erreicht. Saunaanwendungen eignen sich daher auch nicht zum passiven Aufwärmen im Zusammenhang mit Sport vor Trainings- oder Wettkampfsituationen. Eine belastungsarme Durchwärmung der Körperschale - ein Ausgleich der Isothermen auf $37^{\circ} \mathrm{C}-$ ist nicht das Ziel von Saunaanwendungen.

\section{Infrarotkabinen - Durchwärmungsanwendungen}

Mithilfe von Durchwärmungsanwendungen soll auch in der Körperschale (Muskulatur, Binde- und Fettgewebe, Knochen, Gelenke, Sehnen) eine Temperatur von 36-37 ${ }^{\circ} \mathrm{C}$ erreicht werden, ohne im Körperkern künstliches Fieber zu erzeugen. Ausgehend von der Gewebetemperatur $\left(25-34{ }^{\circ} \mathrm{C}\right)$ ist in der Körperschale damit eine Temperatursteigerung um $5-10{ }^{\circ} \mathrm{C}$ möglich. Der Stoffwechsel wird um den Faktor 2-3 gesteigert und die Gewebeversorgung verbessert. Im Verbund mit der gesteigerten Durchblutung werden Effekte erreicht, die man mit den Begriffen «Entschlackung» und «Entgiftung» des Gewebes umschreiben kann. Die Schweissbildung unterstützt die Entgiftung des Organismus in grösserem Umfang.

Die Erkenntnis, dass mit Wärmeanwendungen oberhalb der TNZ eine belastungsarme Durchwärmung kaum zu erreichen ist, hat in Japan und Korea zur Entwicklung der sogenannten Waon-Therapie geführt [102-108].

Zur Waon-Therapie kommen Infrarotwärmekabinen mit Lufttemperaturen von $50-60{ }^{\circ} \mathrm{C}$ zum Einsatz. Die Patienten werden jedoch nur für 10-15 min exponiert und anschliessend für weitere 30-40 min mit einer Wärmestaudecke versorgt. Dadurch wird im Anschluss an die initiale, kurze Wärmeexposition ein Temperaturausgleich über den Körperkern, die Körperschale und die äussere Körperschale erreicht.

\section{Wärmeanwendungen innerhalb der Thermoneutralzone}

Eine andere Möglichkeit besteht in der Nutzung der TNZ. Wird unter diesen Bedingungen $\left(27-37^{\circ} \mathrm{C}\right)$ ein Hautareal (z.B. Rückenbereich) von nicht mehr als ca. 10-12\% der Hautoberfläche erwärmt, so vermischt sich im zentralen Kreislauf $90 \%$ thermoneutrales mit 10\% erwärmtem Blut. Der Schwellenwert der thermosensiblen Strukturen im Thorax wird nicht erreicht. Der Zustrom des erwärmten Blutes zum Körperinnern wird zugelassen, da die Wärme zur Erhaltung der Kerntemperatur schrittweise (Verschiebung der Isothermen) über das Blut von «innen 


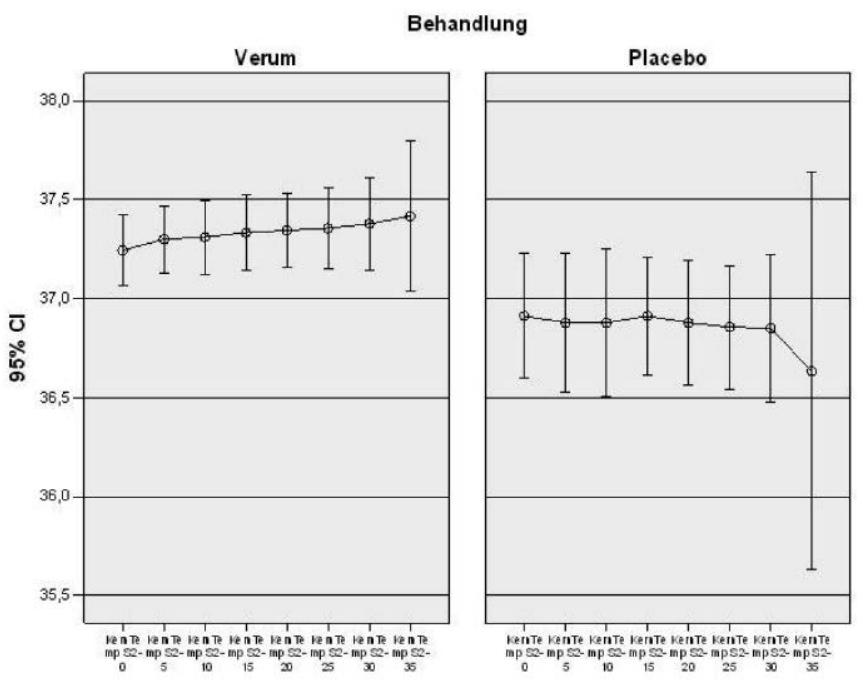

Abb. 5. Änderungen der Körperkerntemperatur in Abhängigkeit von der Expositionsdauer (links: TNZ-Prinzip; rechts: konventionelle IR-Kabine.

heraus» in der Körperschale transferiert werden kann [4, 109]. Die Herzkreislaufbelastung bleibt gering.

In der Praxis bedeutet dies, dass mit dem Einsetzen der Wärmeapplikation der Zustrom von erwärmtem Blut in den Kern aufrechterhalten bleibt. Die KKT steigt leicht und kontinuierlich von Beginn an sowie über einen Zeitraum von 30 min um $0,2-0,3{ }^{\circ} \mathrm{C}$. Im Gegensatz dazu bleibt die KKT in der Anwendung, die dem Saunaprinzip folgt, konstant. Die unterschiedlichen Ausgangstemperaturen in beiden Gruppen sind zufällig [110, 111] (Abb. 5).

Dieser Prozess der Zufuhr und Verteilung thermischer Energie kann mit dem Begriff «Tiefenwärme» charakterisiert werden. «Tiefenwärme» ist somit nicht dem Medium des Wärmeübertrags geschuldet, sondern der Ausnutzung physiologischer Wärmeregulationsreaktionen. Infrarotstrahlung ist dabei das geeignete Medium, dem Körper Wärme zuzuführen, da die Wärmeabwehrreaktion der Haut erhalten bleibt - sofern die beschriebenen Grenzen (Hauteigentemperatur) berücksichtigt werden.

\section{Verschiedene Indikationen der Ganzkörperwärmeanwendungen}

Auf Basis der eingangs beschriebenen Effekte lassen sich grob drei Zielrichtungen der Wärmeanwendung zusammenfassen: die Temperaturerhöhung im Gewebe, kutiviszerale Reflexe im Wechsel mit Kälte und Applikation von Wirksubstanzen über die erwärmte Haut $[5,19$, 112].

Bei lokalen oder lokoregionalen Anwendungen ist in erster Linie die thermische Hautbelastung zu berücksichtigen. Die Nutzung von Permeabilitätsänderungen zur transdermalen Applikation von Substanzen muss Bestandteil einer eigenen Übersicht bleiben.
Im Folgenden liegt der Fokus auf Ganzkörperwärmeanwendungen mit geringer Herzkreislaufbelastung, deren Ziel nicht die Implementierung eines künstlichen Fiebers ist. Bei der Literaturrecherche fällt auf, dass sich bis dato keine einheitliche Terminologie zu Ganzkörperwärmeanwendungen etabliert hat. Der Begriff «Sauna» subsumiert Anwendungen mit unterschiedlichen und nicht vergleichbaren Ausgangs- und Verlaufsparametern. Weitere Begriffe sind unter anderem «Thermal Therapy», "Infrared Cabin», «Infrared Rays» und «Hot Temperature».

Obwohl die Wärmeeffekte bei Gewebetemperaturen von unter $38,5^{\circ} \mathrm{C}$ als unspezifisch anzusehen sind, lassen sich dennoch bei verschiedenen Krankheitsbildern lindernde Effekte durch den Wärmeeinsatz zeigen.

Beschränkt man sich auf jene Publikationen, die eine Ganzkörperwärmeanwendung mit geringer Herzkreislaufbelastung (unter $60^{\circ} \mathrm{C}$ Lufttemperatur) untersuchen, können als beispielhafte Auswahl folgende Indikationen genannt werden: chronische Schmerzen [113], chronisches Müdigkeitssyndrom (chronic fatigue) [114, 115], Depression [113], Fibromyalgie [103, 116, 117], rheumatoide Arthritis [118-120], Osteoarthritis [121, 122], Kreuzschmerzen [123-126], Herzkreislauferkrankungen [106, 108, 127-131], arterielle Erkrankungen [132], Ulcera [132a], Bluthochdruck [106, 133-135], arterielle Verschlusskrankheit [132], Sjögren-Syndrom [136], Sklerodermie [137], Diabetes [138-141], Hauterkrankungen [142-145], Asthma [146], allergische Rhinitis [147], Sport [148-150], mit dem Lebensstil verbundene Erkrankungen [151], Infektionen [152], Leishmaniose [153] und Mikrozirkulation [154].

Eine wesentliche Differenzierung der Ganzkörperwärmeanwendungen, ihrer Wirkbereiche und den daraus resultierenden Belastungen ergibt sich aus der Wärmeregulationsreaktion, die durch sie jeweils angestossen wird. Eine Tiefenwirkung der übertragenen thermischen Energie kann erst mithilfe der körpereigenen Wärmeregulation über das Blut erreicht werden.

\section{Schlussfolgerungen}

Ganzkörperwärmeanwendungen wie die Waon-Therapie oder das TNZ-Prinzip, die auch eine Durchwärmung der Körperschale bei geringer Herzkreislaufbelastung erreichen, scheinen in diesem Zusammenhang hinsichtlich ihrer Wirkung und Verträglichkeit den Ganzkörperwärmeanwendungen oberhalb der TNZ überlegen zu sein.

Um dem Körper effizient Wärme über Haut und Schleimhäute zuführen zu können, ist eine gute lokale Hautdurchblutung und funktionierende Wärmeregulation essenziell. Die Art der Wärmeapplikation sollte die Hautdurchblutung und die Wärmeabgabemechanismen möglichst wenig behindern. Infrarotbestrahlungen sind daher zur Wärmeapplikation vorteilhaft. Um während 
der Anwendung die Einhaltung der maximalen Bestrahlungsstärken sicherzustellen, muss der Abstand zum Strahler definiert sein (gegebenenfalls Sitzplatz vorgeben). Die geltenden Grenzwerte der Bestrahlungsstärke von $100 \mathrm{~mW} / \mathrm{cm}^{2}$ (Haut) und $10 \mathrm{~mW} / \mathrm{cm}^{2}$ (Auge) [33] sind in der Praxis - vor allem bei Menschen mit Einschränkungen der Wärmeregulation - vielfach zu hoch. Die Bestrahlungsstärke sollte laufend der Wärmeaufnahmefähigkeit - also der Durchblutungssituation - der Haut angepasst werden [110, 111]. Der Wärmezufluss sollte nur langsam, mit steigender Durchblutung (Wärmeaufnahmefähigkeit der Haut), erhöht werden. Diesen Forderungen werden vor allem Systeme gerecht, die die Bestrahlungs- stärke auf Basis der aktuellen Hauttemperatur laufend automatisch anpassen.

\section{Disclosure Statement}

Die Firma Physiotherm ist seit 2006 Mandant von OP. Das Mandat erstreckt sich auf die medizinisch-wissenschaftliche Beratung.

TZ ist Physiker und seit 2007 Leiter der Abteilung für Forschung und Entwicklung der Firma Physiotherm.

Das Institut von WS war und ist Kooperationspartner im Rahmen klinischer Studien und Forschungsprojekte.

\section{Literatur}

1 Heckel M: Ganzkörperhyperthermie und Fiebertherapie. Stuttgart, Hippokrates, 1990.

$>2$ Kleef R, Jonas WB, Knogler W, Stenzinger $\mathrm{W}$ : Fever, cancer incidence and spontaneous remissions. Neuroimmunomodulation 2001; 9:55-64.

3 Kleef R, Hager DE: Fever, Pyrogens and Cancer. Madame Curie Bioscience Database (Internet). Austin, Landes Bioscience, 2000.

4 Koralewski HE, Gunga HC, Kirsch KA: Wärmehaushalt und Temperaturregulation. Berlin, Charité, 2010

5 Fialka-Moser V: Kompendium der physikalischen Medizin und Rehabilitation. Wien/New York, Springer, 2005.

6 Hazlett L, Wu M: Defensins in innate immunity. Cell Tissue Res 2011;343:175-188.

7 Schwalfenberg GK: A review of the critical role of vitamin $\mathrm{D}$ in the functioning of the immune system and the clinical implications of vitamin D deficiency. Mol Nutr Food Res 2011;55:96-108.

8 Hoth M, Rettig J: Grundlagen der Zellphysiologie; in Behrends JC, Bischofberger J, Deutzmann R, et al. (Hrsg): Physiologie, ed 1. Stuttgart, Thieme, 2010, pp 2-20.

9 Pichler WJ, Peter HH: Immunsystem; in Siegenthaler W, Blum HE (Hrsg): Klinische Pathophysiologie, ed 9. Stuttgart, Thieme, 2006, pp 478-552.

10 Das S: Ab- und ausleitende Verfahren; in Saller R, Bachmann RM (Hrsg): Naturheil verfahren und Komplementärmedizin in der Praxis. Balingen, Spitta, 1998.

11 Rosslenbroich BS, et al: Physikalische Therapien und Diagnostik im Überblick; in Bühring M, Kemper FH, Matthiessen PF (Hrsg): Naturheilverfahren und Unkonventionelle Medizinische Richtungen. Berlin, Springer, 1998.

12 Ross GH, Sternquist MC: Methamphetamine exposure and chronic illness in police officers: significant improvement with sauna-based detoxification therapy. Toxicol Ind Health 2012;28:758-768.

$>13$ Kleef R: Hyperthermie und Entgiftung; in Marktl W, Reiter B, Ekmekcioglu C (Hrsg): Säuren-Basen-Schlacken. Wien, Springer 2007, pp 133-144.
14 Vaupel P: Wärmehaushalt; in Thews G, Vaupel P (Hrsg): Vegetative Physiologie. Heidelberg, Springer, 2006, pp 322-343.

15 Heine H: Lehrbuch der biologischen Medizin. Stuttgart, Hippokrates, 1997.

16 Lang F, Lang P: Energie- und Wärmehaushalt; in Lang F, Lang P (Hrsg): Basiswissen Physiologie. Heidelberg, Springer, 2007, pp 181-190.

17 Grissmer S: Energie- und Wärmehaushalt; Physiologie. Stuttgart, Thieme, 2010, pp 512-531.

18 Koralewski HE: Energiehaushalt und Temperaturregulation. Berlin, Charité, 2006.

19 Brock FE: Hydro- und Thermotherapie; in Bühring M, Kemper FH, Matthiessen PF (Hrsg): Naturheilverfahren und Unkonventionelle Medizinische Richtungen. Berlin, Springer, 2003.

20 Habash RW, Bansal R, Krewski D, Alhafid HT: Thermal therapy, part 1: an introduction to thermal therapy. Crit Rev Biomed Eng 2006;34:459-489.

21 Lehmann JF, Silverman DR, Baum BA, Kirk NL, Johnston VC: Temperature distributions in the human thigh, produced by infrared, hot pack and microwave applications. Arch Phys Med Rehabil 1966;47:291299.

22 Lehmann P: Photodermatosen; in BraunFalco O, Plewig G, Wolff HH, Burgdorf WHC, Landthaler M (Hrsg): Dermatologie und Venerologie, ed 5. Heidelberg, Springer, 2005, pp 545-570.

23 Rohde J: Physikalische Therapien: Hydround Thermotherapie; in Augustin M, Schmiedel V (Hrsg): Praxisleitfaden Naturheilkunde, ed 1. Neckarsulm, Jungjohann, 1993, pp 163-177.

24 Siragusa M, Schepis C, Palazzo R, Fabrizi G, Guarneri B, Del Gracco S, Spada RS, Ferri R: Skin pathology findings in a cohort of 1500 adult and elderly subjects. Int J Dermatol 1999;38:361-366.

25 Papp A: The first 1000 patients treated in Kuopio University Hospital Burn Unit in Finland. Burns 2009;35:565-571.

26 Alotaibi LI, Hammadi AA: Erythema ab igne: Follow-up. Emedicine. http://emedicine. medscape.com/article/1087535-overview. Updated 2012.

27 Howe NR, Bader RS: Erythema ab igne; in Demis DJ (ed): Clinical Dermatology. Philadelphia, Lippincott-Raven, 1998, pp 7-8.

28 Wienert V, Sick H, zur Muhlen J: Local thermal stress tolerance of human skin (article in German). Anasth Intensivther Notfallmed 1983;18:88-90.

29 Kenny GP, Journeay WS: Human thermoregulation: separating thermal and non thermal effects on heat loss. Front Biosci 2010;15:259-290.

30 Hodges GJ, Johnson JM: Adrenergic control of the human cutaneous circulation. Appl Physiol Nutr Metab 2009;34:829-839.

31 Jonas HS, et al: Automatisierte, individuelle Ganzkörperüberwärmung auf Basis der Niedertemperaturinfrarottechnologie zur Erhöhung der Sicherheit der Anwendung und Effizienzsteigerung bei besonderen Personengruppen unter Kontrolle physiologischer Parameter, 2011.

32 Lenhardt R: The effect of anesthesia on body temperature control. Front Biosci (Schol Ed) 2010;2:1145-1154.

33 International Commission on Non-Ionizing Radiation Protection: ICNIRP statement on far infrared radiation exposure. Health Phys 2006;91:630-645.

34 Altintas MA, Altintas AA, Guggenheim M, Niederbichler AD, Knobloch K, Vogt PM: In vivo evaluation of histomorphological alterations in first-degree burn injuries by means of confocal-laser-scanning micros copy - more than 'virtual histology?'. J Burn Care Res 2009;30:315-320.

35 Proksch E, Jensen JM, Crichton-Smith A, Fowler A, Clitherow J: Rationale Behand lung von Patienten mit Verbrennungen 1. Grades. Hautarzt 2007;58:604-610.

36 Kagan RJ, Smith SC: Evaluation and treatment of thermal injuries. Dermatol Nurs 2000;12:334-344, 347.

37 Runitz K, Jensen TH: Heat stroke and burns resulting from use of sauna (article in Danish). Ugeskr Laeger 2009;171:305-306. 
-38 Tan S, Bertucci V: Erythema ab igne: an old condition new again. CMAJ 2000;162:7778.

-39 Cavallari V, Cicciarello R, Torre V, Gagliardi ME, Albiero F, Palazzo R, Siragusa M, Schipis C: Chronic heat-induced skin lesions (erythema ab igne): ultrastructural studies. Ultrastruct Pathol 2001;25:93-97.

-40 de Jong EM, Koopman RJ, van de Kerkhof PC: Erythema ab igne (article in Dutch) Ned Tijdschr Geneeskd 1995;139:192-194.

41 Wani I: Kangri cancer. Surgery 2010;147: 586-588.

42 Butler ML: Erythema ab igne, a sign of pancreatic disease. Am J Gastroenterol 1977; 67:77-79.

43 Dellavalle RP, Gillum P: Erythema ab igne following heating/cooling blanket use in the intensive care unit. Cutis 2000;66:136-138.

$\checkmark 4$ Chatterjee S: Erythema ab igne from prolonged use of a heating pad. Mayo Clin Proc 2005;80:1500.

-45 Radmanesh M: Erythema ab igne following sauna belt use for abdominal obesity and cellulite. Int J Dermatol 2009;48:94-95.

-46 Donohue KG, Nahm WK, Badiavas E, Li L, Pedvis-Leftick A: Hot pop brown spot: erythema ab igne induced by heated popcorn J Dermatol 2002;29:172-173.

-47 Helm TN, Spigel GT, Helm KF: Erythema ab igne caused by a car heater. Cutis 1997; 59:81-82

$\checkmark 48$ Andersen F: Laptop-thighs - laptop-induced erythema ab igne (article in Danish). Ugeskr Laeger 2010;172:635.

-49 Lin SJ, Hsu CJ, Chiu HC: Erythema ab igne caused by frequent hot bathing. Acta Derm Venereol 2002;82:478-479.

-50 Darvin ME, Haag S, Meinke M, Zastrow L, Sterry W, Lademann J: Radical production by infrared A irradiation in human tissue. Skin Pharmacol Physiol 2010;23:40-46.

- 51 Calles C, Schneider M, Macaluso F, Benesova T, Krutmann J, Schroeder P: Infrared A ra diation influences the skin fibroblast transcriptome: mechanisms and consequences. J Invest Dermatol 2010;130:1524-1536.

- 52 Bergamaschi A, Grandi C, D’Addato M Di Carlo V, Russo A: Health risks from infrared emissions from radiant tube heaters in the workplace (article in Italian). G Ital Med Lav 1995; 17:41-49.

53 Page EH, Shear NH: Temperature-dependent skin disorders. J Am Acad Dermatol 1988;18:1003-1019.

54 Cho S, Shin MH, Kim YK, Seo JE, Lee YM, Park $\mathrm{CH}$, Chung JH: Effects of infrared ra diation and heat on human skin aging in vivo. J Investig Dermatol Symp Proc 2009; 14:15-19.

55 Beleznay K, Humphrey S, Au S: Erythema ab igne. CMAJ 2010;182:E228.

56 Seegenschmiedt MH, Klautke G, Walther E, Feldmann HJ, Katalinic A, Stuschke M, von Lieven H, Vaupel P: Wassergefilterte Infrarot-A-Hyperthermie, kombiniert mit Radiotherapie bei forgeschrittenen und rezidivierten Tumoren. Erste Ergebnisse einer multizentrischen Phase-I/II-Studie. Strahlenther Onkol 1996;172:475-484.
57 Deutsche Physikalische Gesellschaft: Das elektromagnetische Spektrum. Deutsche Physikalische Gesellschaft e.V., 2009, 2012. www.weltderphysik.de/gebiete/atomel elektromagnetisches-spektrum.

58 Carpenter DO: Electromagnetic fields and cancer: the cost of doing nothing. Rev Environ Health 2010;25:75-80

59 Flory R, Ametepe J, Bowers B: A randomized, placebo-controlled trial of bright light and high-density negative air ions for treatment of seasonal affective disorder. Psychiatry Res 2010;177:101-108.

60 Griefahn B, Kunemund C, Blaszkewicz M, Lerchl A, Degen GH: Effects of electromagnetic radiation (bright light, extremely lowfrequency magnetic fields, infrared radiation) on the circadian rhythm of melatonin synthesis, rectal temperature, and heart rate. Ind Health 2002;40:320-327.

61 Lask G, Fournier N, Trelles M, Elman M, Scheflan M, Slatkine M, Naimark J, Harth Y: The utilization of nonthermal blue (405-425 $\mathrm{nm})$ and near infrared (850-890 nm) light in aesthetic dermatology and surgery - a multicenter study. J Cosmet Laser Ther 2005;7:163-170.

62 Ankri R, Lubart R, Taitelbaum H: Estimation of the optimal wavelengths for laserinduced wound healing. Lasers Surg Med 2010;42:760-764.

63 Schuz J, Elliott P, Auvinen A, Kromhout H, Poulsen AH, Johansen C, Olsen JH, Hillert L, Feychting M, Fremling K, Toledano M, Heinavaara S, Slottje $P$, Vermeulen R, Ahlbom A: An international prospective cohort study of mobile phone users and health (Cosmos): design considerations and enrolment. Cancer Epidemiol 2011;35:37-43.

64 Degrave E, Meeusen B, Grivegnee AR, Boniol M, Autier P: Causes of death among Belgian professional military radar operators: a 37-year retrospective cohort study. Int J Cancer 2009;124:945-951.

65 Richter ED, Berman T, Levy O: Brain cancer with induction periods of less than 10 years in young military radar workers. Arch Environ Health 2002;57:270-272.

66 Alekseev SI, Gordiienko OV, Ziskin MC: Reflection and penetration depth of millimeter waves in murine skin. Bioelectromagnetics 2008;29:340-344

67 Alekseev SI, Ziskin MC: Local heating of human skin by millimeter waves: a kinetics study. Bioelectromagnetics 2003;24:571-581.

68 Grissmer S: Arbeits-, Sport- und Leistungsphysiologie; Physiologie. Stuttgart, Thieme, 2010, pp 534-554.

69 Dover JS, Phillips TJ, Arndt KA: Cutaneous effects and therapeutic uses of heat with emphasis on infrared radiation. J Am Acad Dermatol 1989;20:278-286.

70 Weingarten MS, Papazoglou ES, Zubkov L, Zhu L, Neidrauer M, Savir G, Peace K, Newby JG, Pourrezaei K: Correlation of near infrared absorption and diffuse reflectance spectroscopy scattering with tissue neovascularization and collagen concentration in a diabetic rat wound healing model. Wound Repair Regen 2008;16:234-242.
71 Anforderungen an öffentliche und gewerbliche Saunaanlagen, Infrarotkabinen, Dampfund sonstige Wärmekammern. Teil 2: Planung und Betrieb von Infrarotkabinen. 2010 OENORM M 6219-2:2010-03-01

71a Charkoudian N: Mechanisms and modifiers of reflex induced cutaneous vasodilation and vasoconstriction in humans. J Appl Physiol (1985) 2010;109:1221-1228.

72 Pujol JA, Lecha M: Photoprotection in the infrared radiation range. Photodermatol Photoimmunol Photomed 1992;9:275-278.

73 Zastrow L, Groth N, Klein F, Kockott D, Lademann J, Renneberg R, Ferrero L: The missing link - light-induced (280-1,600 nm) free radical formation in human skin. Skin Pharmacol Physiol 2009;22:31-44.

74 Hsu YH, Chen YC, Chen TH, Sue YM, Cheng TH, Chen JR, Chen CH: Far-infrared therapy induces the nuclear translocation of PLZF which inhibits VEGF-induced proliferation in human umbilical vein endothelial cells. PLoS One 2012;7:e30674.

75 Schroeder P, Calles C, Benesova T, Macaluso F, Krutmann J: Photoprotection beyond ultraviolet radiation - effective sun protection has to include protection against infrared a radiation-induced skin damage. Skin Pharmacol Physiol 2010;23:15-17.

76 Schroeder P, Haendeler J, Krutmann J: The role of near infrared radiation in photoaging of the skin. Exp Gerontol 2008;43:629-632.

77 Hoffmann G: Water-filtered infrared-A (Wira) in acute and chronic wounds. GMS Krankenhhyg Interdiszip 2009;4:Doc12.

78 Esnouf A, Wright PA, Moore JC, Ahmed S: Depth of penetration of an $850 \mathrm{~nm}$ wavelength low level laser in human skin. Acupunct Electrother Res 2007;32:81-86.

79 Kolari PJ, Airaksinen O: Poor penetration of infra-red and helium neon low power laser light into the dermal tissue. Acupunct Electrother Res 1993;18:17-21.

80 Kolari PJ: Penetration of unfocused laser light into the skin. Arch Dermatol Res 1985;277:342-344.

81 Bruls WA, Slaper H, van der Leun JC, Berrens L: Transmission of human epidermis and stratum corneum as a function of thickness in the ultraviolet and visible wavelengths. Photochem Photobiol 1984;40:485-494.

82 Piazena H, Kelleher DK: Effects of infraredA irradiation on skin: discrepancies in published data highlight the need for an exact consideration of physical and photobiological laws and appropriate experimental settings. Photochem Photobiol 2010;86:687705.

83 Meglinski IV, Matcher SJ: Computer simulation of the skin reflectance spectra. Comput Methods Programs Biomed 2003;70:179186.

84 Brancaleon L, Bamberg MP, Sakamaki T, Kollias N: Attenuated total reflectionfourier transform infrared spectroscopy as a possible method to investigate biophysical parameters of stratum corneum in vivo. J Invest Dermatol 2001;116:380-386.

85 Azzolini C, Gobbi PG, Brancato R, Bosi L, Gallo D, Zelada M, Patelli F: Interaction be tween infrared radiation and vitreous substitutes. Arch Ophthalmol 1997;115:899-903. 
86 Arimoto H, Egawa M, Yamada Y: Depth profile of diffuse reflectance near-infrared spectroscopy for measurement of water content in skin. Skin Res Technol 2005; 11:27-35.

-87 Milani V, Pazos M, Issels RD, Buecklein V, Rahman S, Tschoep K, Schaffer P, Wilkowski R, Duehmke E, Schaffer M: Radiochemotherapy in combination with regional hyperthermia in preirradiated patients with recurrent rectal cancer. Strahlenther Onkol 2008;184:163-168.

-88 Issels RD: Hyperthermia adds to chemotherapy. Eur J Cancer 2008;44:2546-2554.

89 Hellige G, Becker G, Hahn G: Temperaturverteilung und Eindringtiefe wassergefilterter Infrarot-A-Strahlung; in Vaupel P, Krüger W (Hrsg): Wärmetherapie mit wassergefilterter Infrarot-A-Strahlung. Stuttgart, Hippokrates, 1995, pp 63-79.

89a Johnson JM, Kellogg DL Jr: Thermoregulatory and thermal control in the human cutaneous circulation. Front Biosci (Schol Ed) 2010;2:825-853.

90 Gekle M, Singer D: Thermoregulation und Wärmehaushalt; in Klinke R, Pape HC, Kurtz A, Silbernagel S (Hrsg): Physiologie. Stuttgart, Thieme, 2009, pp 500-515.

-91 Sohar E, Shoenfeld Y, Shapiro Y, Ohry A Cabili S: Effects of exposure to Finnish sauna. Isr J Med Sci 1976;12:1275-1282.

-91a Gonzalez-Alonso J: Human thermoregulation and the cardiovascular system. Exp Physiol 2012;97:340-346.

-92 Schlader ZJ, Prange HD, Mickleborough TD, Stager JM: Characteristics of the control of human thermoregulatory behavior. Physiol Behav 2009;98:557-562.

$>93$ Kauppinen K: Sauna, shower, and ice water immersion. Physiological responses to brief exposures to heat, cool, and cold. Part III. Body temperatures. Arctic Med Res 1989;48:75-86.

-94 Kauppinen K: Sauna, shower, and ice water immersion. Physiological responses to brief exposures to heat, cool, and cold. Part II. Circulation. Arctic Med Res 1989;48:64-74.

$>95$ Kauppinen K: Sauna, shower, and ice water immersion. Physiological responses to brief exposures to heat, cool, and cold. Part I. Body fluid balance. Arctic Med Res 1989;48:55-63.

$\checkmark 96$ Shoenfeld Y, Sohar E, Ohry A, Shapiro Y: Heat stress: comparison of short exposure to severe dry and wet heat in saunas. Arch Phys Med Rehabil 1976;57:126-129.

$\checkmark 9$ Schoenfeld Y, Shapira Y, Sohar E: Sauna and its influence on the human body (article in Hebrew). Harefuah 1977;92:512513.

$\$ 98$ Shoenfeld Y, Udassin R, Shapiro Y, Ohri A, Sohar E: Age and sex difference in response to short exposure to extreme dry heat. J Appl Physiol Respir Environ Exerc Physiol 1978;44:1-4.

$\checkmark 99$ Rodhe A, Eriksson A: Sauna deaths in Sweden, 1992-2003. Am J Forensic Med Pathol 2008;29:27-31.

100 Livingston R: Medical risks and benefits of the sweat lodge. J Altern Complement Med 2010;16:617-619.
101 Kukkonen-Harjula K, Kauppinen K: Health effects and risks of sauna bathing. Int J Circumpolar Health 2006;65:195-205.

102 Kihara T, Miyata M, Fukudome T, Ikeda Y, Shinsato T, Kubozono T, Fujita S, Kuwahata S, Hamasaki S, Torii H, Lee S, Toda H, Tei C: Waon therapy improves the prognosis of patients with chronic heart failure. J Cardiol 2009;53:214-218.

103 Matsushita K, Masuda A, Tei C: Efficacy of Waon therapy for fibromyalgia. Intern Med 2008;47:1473-1476.

104 Iiyama J, Matsushita K, Tanaka N, Kawahira K: Effects of single low-temperature sauna bathing in patients with severe motor and intellectual disabilities. Int J Biometeorol 2008;52:431-437.

105 Tei C: Waon therapy: soothing warmth therapy. J Cardiol 2007;49:301-304.

106 Miyata M, Tei C: Waon therapy for cardiovascular disease: innovative therapy for the 21st century. Circ J 2010;74:617-621.

107 Fujita S, Ikeda Y, Miyata M, Shinsato T, Kubozono T, Kuwahata S, Hamada N, Miyauchi T, Yamaguchi T, Torii H, Hamasaki S, Tei C: Effect of Waon therapy on oxidative stress in chronic heart failure. Circ J 2011;75:348-356.

108 Sobajima M, Nozawa T, Ihori H, Shida T, Ohori T, Suzuki T, Matsuki A, Yasumura S, Inoue $\mathrm{H}$ : Repeated sauna therapy improves myocardial perfusion in patients with chronically occluded coronary artery-related ischemia. Int J Cardiol 2013;167:237-243.

109 Ulmer HV: Arbeits- und Umweltphysiologie; in Schmidt RF, Thews G (Hrsg): Physiologie des Menschen. Berlin, Springer, 1987, pp 683-716.

110 Kleef R, Zimmermann A, Pecher O: Explorative Untersuchungen zur Niedertemperatur-Infrarot-Technik (Physiotherm). Thermoregulation und Belastung der Haut. Sicherheitsstudie. 2007. www.physiothermkaiserslautern.de/gesundheit-infrarot/ forschung-studien/die-grundlagenstudie/\#1.

111 Kleef R, Schmolz M, Maerz R, Pecher O, Saller R: Explorative Untersuchungen zur Niedertemperatur-Infrarot-Technik (Physiotherm) mit ILCS (instant leukocyte culture system) über physiologische und immunmodulierende Effekte. Eine Pilotstudie der Phase I. 2007. www.physiothermkaiserslautern.de/gesundheit-infrarot/ forschung-studien/die-grundlagenstudie/\#0.

112 Fialka-Moser V: Therapeutische und physiologische Grundlagen der Hydrotherapie; in Fialka-Moser V (Hrsg): Hydrotherapie. München, Richard Pflaum, 2009, pp 9-71.

113 Masuda A, Nakazato M, Kihara T, Minagoe S, Tei C: Repeated thermal therapy diminishes appetite loss and subjective complaints in mildly depressed patients. Psychosom Med 2005;67:643-647.

114 Masuda A, Kihara T, Fukudome T, Shinsato T, Minagoe S, Tei C: The effects of repeated thermal therapy for two patients with chronic fatigue syndrome. J Psychosom Res 2005;58:383-387.

115 Masuda A, Munemoto T, Tei C: A new treatment: thermal therapy for chronic fatigue syndrome (article in Japanese). Nihon Rinsho 2007;65:1093-1098.
116 Fioravanti A, Bellisai B, Capitani S, Manica P, Paolazzi G, Galeazzi M: Phytothermotherapy: a possible complementary therapy for fibromyalgia patients. Clin Exp Rheumatol 2009;27:S29-32.

117 Isomaki H: The sauna and rheumatic diseases. Ann Clin Res 1988;20:271-275.

118 Berliner MN: Thermotherapy in rheumatic diseases (article in German). Z Arztl Fortbild Qualitatssich 1999;93:331-334.

119 Oosterveld FG, Rasker JJ, Floors M, Landkroon R, van Rennes B, Zwijnenberg J, van de Laar MA, Koel GJ: Infrared sauna in patients with rheumatoid arthritis and ankylosing spondylitis. A pilot study showing good tolerance, short-term improvement of pain and stiffness, and a trend towards long-term beneficial effects. Clin Rheumatol 2009;28:29-34.

120 Robinson V, Brosseau L, Casimiro L, Judd M, Shea B, Wells G, Tugwell P: Thermotherapy for treating rheumatoid arthritis. Cochrane Database Syst Rev 2002;CD002826.

121 Brosseau L, Yonge KA, Robinson V, Marchand S, Judd M, Wells G, Tugwell P: Thermotherapy for treatment of osteoarthritis. Cochrane Database Syst Rev 2003; CD004522.

122 Takahashi KA, Tonomura H, Arai Y, Terauchi R, Honjo K, Hiraoka N, Hojo T, Kunitomo T, Kubo T: Hyperthermia for the treatment of articular cartilage with osteoarthritis. Int J Hyperthermia 2009; 25:661-667.

123 Gale GD, Rothbart PJ, Li Y: Infrared therapy for chronic low back pain: a randomized, controlled trial. Pain Res Manag 2006;11: 193-196.

124 McCarberg BH: Acute back pain: benefits and risks of current treatments. Curr Med Res Opin 2010;26:179-190.

125 Shakoor MA, Rahman MS, Moyeenuzzaman M: Effects of deep heat therapy on the patients with chronic low back pain Mymensingh Med J 2008;17:S32-38.

126 Kumar S, Sharma VP, Shukla R, Dev R: Comparative efficacy of two multimodal treatments on male and female sub-groups with low back pain (part II). J Back Musculoskelet Rehabil 2010;23:1-9.

127 Beever R: Far-infrared saunas for treatment of cardiovascular risk factors: summary of published evidence. Can Fam Physician 2009;55:691-696.

128 Kihara T, Biro S, Ikeda Y, Fukudome T, Shinsato T, Masuda A, Miyata M, Hamasaki S, Otsuji Y, Minagoe S, Akiba S, Tei C: Effects of repeated sauna treatment on ventricular arrhythmias in patients with chronic heart failure. Circ J 2004;68:11461151.

129 Lin CC, Liu XM, Peyton K, Wang H, Yang WC, Lin SJ, Durante W: Far infrared therapy inhibits vascular endothelial in flammation via the induction of heme oxygenase-1. Arterioscler Thromb Vasc Biol 2008;28:739-745.

130 Tei C: Thermotherapy for heart failure in the aged (article in Japanese). Nippon Naika Gakkai Zasshi 2006;95:426-431. 
131 Kuwahata S, Miyata M, Fujita S, Kubozono T, Shinsato T, Ikeda Y, Hamasaki S, Kuwaki T, Tei C: Improvement of autonomic nervous activity by Waon therapy in patients with chronic heart failure. J Cardiol 2011;57:100-106.

$>132$ Tei C, Shinsato T, Miyata M, Kihara T, Hamasaki S: Waon therapy improves peripheral arterial disease. J Am Coll Cardiol 2007;50:2169-2171.

132a Nishijima C, Inaoki M: Digital ulcer in systemic sclerosis successfully treated with Waon therapy. Int J Rheum Dis 2013;16:102104.

133 Meffert B, Hochmuth O, Steiner M, Scherf HP, Meffert H: Effects of a multiple mild infra-red-A induced hyperthermia on central and peripheral pulse waves in hypertensive patients. Med Biol Eng Comput 1991;29:NS45-NS48.

134 Scherf HP, Meffert H, Baumler H, Dittmann K, Siewert H, Strangfeld D, Winterfeld HJ, Hecht HC, Schuhmann E, Sonnichsen $\mathrm{N}$ : Wirkung einer einmaligen Infrarot-A-Hyperthermie auf Körpertemperatur, Herzfrequenz, Blutdruck und Blutviskosität bei Gesunden und Patienten mit arterieller Hypertonie der Stadien I und II. Dermatol Monatsschr 1989;175:733740 .

135 Umehara M, Yamaguchi A, Itakura S, Suenaga M, Sakaki Y, Nakashiki K, Miyata M, Tei C: Repeated Waon therapy improves pulmonary hypertension during exercise in patients with severe chronic obstructive pulmonary disease. J Cardiol 2008;51:106-113.

136 Tei C, Orihara FK, Fukudome T: Remarkable efficacy of thermal therapy for Sjögren syndrome. J Cardiol 2007;49:217-219.
137 Meffert H, Buchholtz I, Brenke A: Milde Infrarot-A-Hyperthermie zur Behandlung der systemischen Sklerodermie. Dermatol Monatsschr 1990;176:683-686.

138 Beever R: The effects of repeated thermal therapy on quality of life in patients with type II diabetes mellitus. J Altern Complement Med 2010;16:677-681.

139 Koivisto VA: Influence of heat on insulin absorption: different effects on amorphous and soluble insulins. Acta Diabetol Lat 1983;20:175-178.

140 Koivisto VA: Various influences on insulin absorption. Neth J Med 1985;28(suppl 1): 25-28.

141 McCarty MF, Barroso-Aranda J, Contreras F: Regular thermal therapy may promote insulin sensitivity while boosting expression of endothelial nitric oxide synthase effects comparable to those of exercise training. Med Hypotheses 2009;73:103-105.

142 Berliner MN, Maurer AI: Effect of different methods of thermotherapy on skin microcirculation. Am J Phys Med Rehabil 2004; 83:292-297.

143 Kim MS, Kim YK, Cho KH, Chung JH: Infrared exposure induces an angiogenic switch in human skin that is partially mediated by heat. Br J Dermatol 2006;155: 1131-1138.

144 Meffert H: Again about the therapeutic effect of hyperthermia in psoriasis (article in German). Dermatol Monatsschr 1990; 176:795-796.

145 Perminova EB, Gridneva TD: EHF-therapy and IR radiation in combined treatment of children suffering from atopic dermatitis (article in Russian). Vopr Kurortol Fizioter Lech Fiz Kult 2005;24-26.
146 Meyer R: Bronchial asthma - heat treatment permits patients to breathe easier (article in German). Pneumologie 2007; 61:624.

$147 \mathrm{Hu}$ KH, Li WT: Clinical effects of far-infrared therapy in patients with allergic rhinitis. Conf Proc IEEE Eng Med Biol Soc 2007;2007:1479-1482.

148 Brock Symons T, Clasey JL, Gater DR, Yates JW: Effects of deep heat as a preventative mechanism on delayed onset muscle soreness. J Strength Cond Res 2004;18:155-161.

49 Hedley AM, Climstein M, Hansen R: The effects of acute heat exposure on muscular strength, muscular endurance, and muscular power in the euhydrated athlete. J Strength Cond Res 2002;16:353358.

150 Racinais S, Chamari K, Hachana Y, Bartagi $\mathrm{Z}$, Blonc S, Hue O: Effect of an acute hot and dry exposure in moderately warm and humid environment on muscle performance at different times of day. Int J Sports Med 2006;27:49-54.

151 Biro S, Masuda A, Kihara T, Tei C: Clinical implications of thermal therapy in lifestylerelated diseases. Exp Biol Med (Maywood) 2003;228:1245-1249.

152 Ellis SL, Finn P, Noone M, Leaper DJ: Eradication of methicillin-resistant Staphylococcus aureus from pressure sores using warming therapy. Surg Infect (Larchmt) 2003;4:53-55.

153 Junaid AJ: Treatment of cutaneous leishmaniasis with infrared heat. Int J Dermatol 1986;25:470-472.

154 Shinsato T, Miyata M, Kubozono T, Ikeda Y, Fujita S, Kuwahata S, Akasaki Y, Hamasaki S, Fujiwara H, Tei C: Waon therapy mobilizes CD34+ cells and improves peripheral arterial disease. J Cardiol 2010;56:361-316. 